Zhao, J.; Özgen-Xian, I.; Liang, D.; Wang, T.; Hinkelmann, R.

\title{
An improved multislope MUSCL scheme for solving shallow water equations on unstructured grids
}

Journal article | Accepted manuscript (Postprint)

This version is available at https://doi.org/10.14279/depositonce-8415

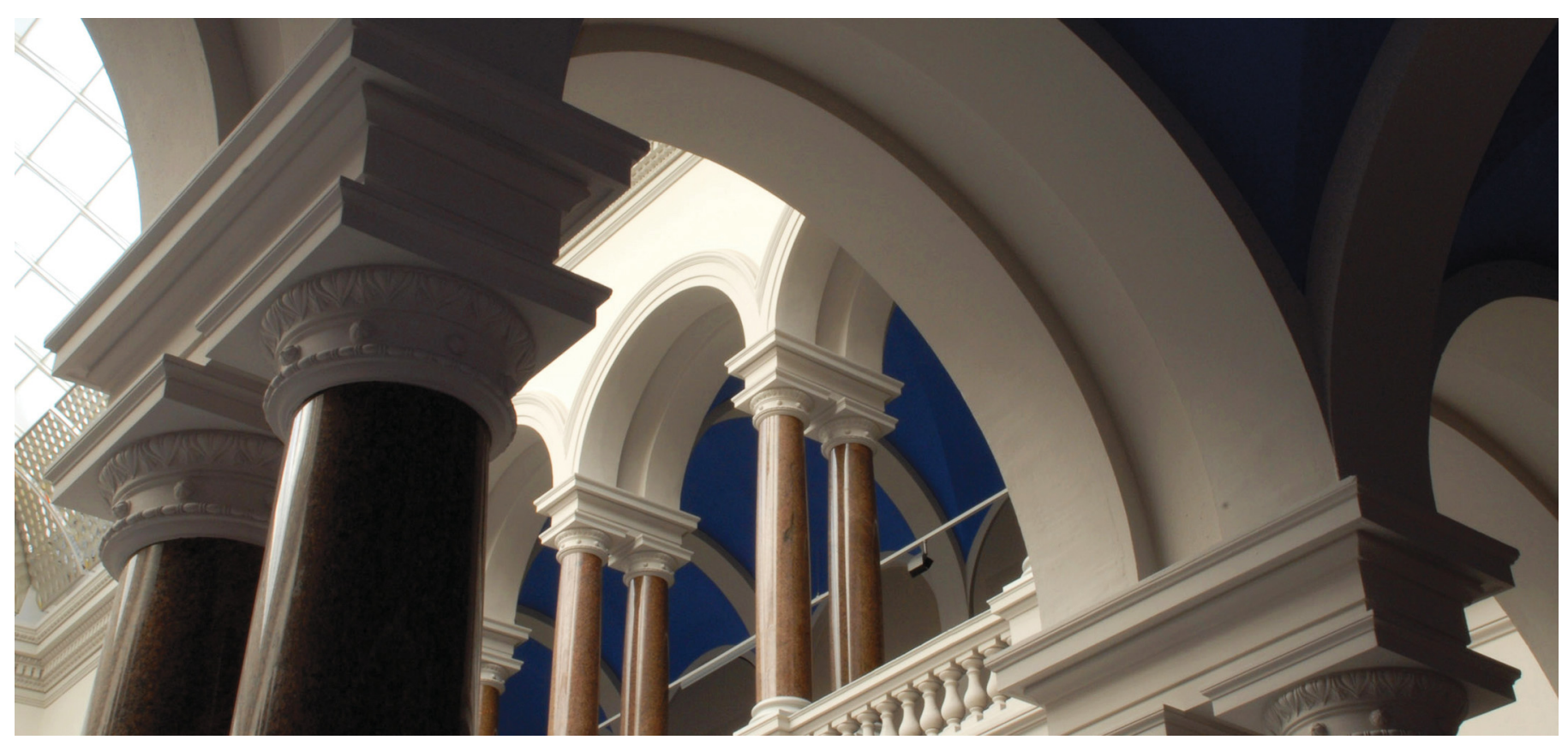

Zhao, J., Özgen-Xian, I., Liang, D., Wang, T., \& Hinkelmann, R. (2019). An improved multislope MUSCL scheme for solving shallow water equations on unstructured grids. Computers \& Mathematics with Applications, 77(2), 576-596. https://doi.org/10.1016/j.camwa.2018.09.059 


\title{
An improved multislope MUSCL scheme for solving shallow water equations on unstructured grids
}

\author{
Jiaheng Zhao ${ }^{\mathrm{a}}$, Ilhan Özgen-Xian ${ }^{\mathrm{a}}$, Dongfang Liang ${ }^{\mathrm{b}}$, Tian Wang ${ }^{\mathrm{a}, \mathrm{c}}$, \\ Reinhard Hinkelmann ${ }^{\mathrm{a}}$ \\ ${ }^{a}$ Chair of Water Resources Management and Modeling of Hydrosystems, Technische \\ Universität Berlin, Germany \\ ${ }^{b}$ Department of Engineering, University of Cambridge, Cambridge, UK \\ ${ }^{c}$ Institute of Water Resources and Hydro-Electric Engineering, Xi'an University of \\ Technology, Xi'an 710048, China
}

\begin{abstract}
This paper describes an improved vector manipulation multislope monotone upstream-centred scheme for conservation laws (MUSCL) reconstruction for solving the shallow water equations on unstructured grids. This improved MUSCL reconstruction method includes a bigger stencil for the interpolation and saves time for determining the geometric relations compared to the original vector manipulation method, so it is computationally more efficient and straightforward to implement. Four examples involving an analytical solution, laboratory experiments and field-scale measurements are used to test the performance of the proposed scheme. It has been proven that the proposed scheme can provide comparable accuracy and higher efficiency compared to the original vector manipulation method. With the increasing of the number of cells, the advantage of the proposed scheme becomes more apparent.
\end{abstract}

Keywords: finite volume method, MUSCL scheme, shallow water, total variation diminishing, unstructured grids

Preprint submitted to Computers \&3 Mathematics with Applications September 23, 2018 


\section{Highlights}

2 1. An improved vector manipulation multislope MUSCL method is pre$3 \quad$ sented on unstructured grids with the aim of better accuracy and higher $4 \quad$ efficiency

5 2. The improved scheme includes a bigger stencil for the interpolation and 6 is more straightforward to implement than the original method

$7 \quad 3$. The numerical model shows good agreement with measurements as long $8 \quad$ as the shallow flow assumptions are satisfied

9

10

\section{Introduction}

Monotone upstream-centred scheme for conservation laws (MUSCL) [1] is a well-known approach for achieving high-order accuracy by data reconstruction for solving hyperbolic partial differential equations. In hydrodynamics, many researchers use the MUSCL scheme to solve the two-dimensional shallow water equations (SWEs) due to its monotonicity and high order accuracy (e.g. $[2,3,4,5])$. The MUSCL-type schemes are an extension of the original Godunov scheme [6]. The variable values along the cell edges are extrapolated from the cell centres, and the reconstructed values are stored at the edges to calculate the Riemann flux across the edges. In order to avoid spurious oscillations and produce physically meaningful results, the numerical scheme should be monotonic. The monotonic numerical scheme can be deduced by examining the total variation, which is defined as an over time decreasing summation of the differences between each all adjacent cells. 
Early TVD schemes were derived on structured grids. Directly applying them on unstructured grids often leads to poor results, because the structured grid provides a simple stencil layout for figuring out the upwind and downwind neighbors. On unstructured grids, the upwind and downwind neighbors are often not located along the perpendicular bisector of the edge. This has to be accounted for in MUSCL reconstructions on unstructured grids.

On unstructured grids, TVD MUSCL schemes can be divided into monoslope and multislope methods [2]. The monoslope method was initially presented in [7], which calculates a single slope for the entire cell based on the three immediate neighbors of the cell [8]. The multislope method calculates a slope for each edge based on a three-point stencil. Stencils are set up based on the extrapolation in the upwind direction, and the variable values at the upwind point can be interpolated [9], [4], or set to the value at the cell centre that is closest to the perpendicular bisector of the considered edge. These methods require significant computational effort to determine the upwind cells and the upwind point in the stencil. A poor choice of the upwind point introduces significant numerical errors, even leading to the loss of the TVD property.

As discussed in Hou et al. [5], the multislope method can provide a more efficient and straightforward scheme. Although the multislope method may not provide a piecewise linear slope for the considered cell, the reconstructed values only determine the fluxes across the edges and thus will not influence the conservation law. Therefore, the shape of the reconstructed function inside the cell is not of importance for the FVM [5].

The vector based manipulation method is proposed by Buffard and Clain 
[2], who provide a very straightforward method on complex unstructured grids, especially suitable for multi-dimensional schemes. Based on the idea of Buffard and Clain [2], Hou et al. [5] proposed a new vector based manipulation multislope method. However, in the authors' previous work [8], it was found that their scheme [5] does not include enough downwind information for the calculation of the downwind slopes, which may lead to the wrong interpolation. In order to overcome this problem, Zhao et al. [8] calculates the down-slope value in the downwind direction, which increases the robustness and accuracy. A new multislope MUSCL method is devised in this work to improve the accuracy, which includes more stencil points to maintain the monotonicity of the scheme in different flow conditions.

This study is based on the framework of unstructured Godunov-type cellcentered FVM. The new MUSCL scheme is compared with the scheme in [8] and analytical analyses.

\section{Governing equations and numerical model}

The two-dimensional shallow water equations (SWEs) are derived from the depth-averaged Navier-Stokes equations. They can be written in the conservative vector form as:

$$
\frac{\partial \mathbf{q}}{\partial t}+\frac{\partial \mathbf{f}}{\partial x}+\frac{\partial \mathbf{g}}{\partial y}=\mathbf{s}
$$

with vectors defined as

$$
\mathbf{q}=\left[\begin{array}{c}
h \\
q_{x} \\
q_{y}
\end{array}\right], \quad \mathbf{f}=\left[\begin{array}{c}
q_{x} \\
u q_{x}+g h^{2} / 2 \\
u q_{y}
\end{array}\right], \quad \mathbf{g}=\left[\begin{array}{c}
q_{y} \\
v q_{x} \\
v q_{y}+g h^{2} / 2
\end{array}\right]
$$




$$
\mathbf{s}=\left[\begin{array}{c}
0 \\
-g h \frac{\partial z}{\partial x}-c_{f} u \sqrt{u^{2}+v^{2}} \\
-g h \frac{\partial z}{\partial y}-c_{f} v \sqrt{u^{2}+v^{2}}
\end{array}\right],
$$

where $x$ and $y$ are the Cartesian coordinates, $t$ is time, $\mathbf{q}$ represents the unknown variable vector consisting of $h, q_{x}$ and $q_{y}$ denoting the water depth, unit-width discharges in $x$ - and $y$-direction, respectively. $u, v$ are defined as depth-averaged velocities in $x$ - and $y$-direction, respectively; $\mathbf{f}$ and $\mathbf{g}$ are the flux vectors in $x$ - and $y$-direction, respectively; $\mathbf{s}$ is the source term that includes bed slope and friction contributions, $z$ is the bed elevation and $c_{f}$ is the bed roughness coefficient calculated as $g n^{2} / h^{1 / 3}, g$ is the gravitational acceleration. Viscous and turbulent flux terms are neglected in this equation.

\subsection{Finite volume discretization of SWEs on unstructured grids}

The shallow water equations(SWEs) in Eq. (1) can be integrated over a cell as

$$
\int_{\Omega} \frac{\partial \mathbf{q}}{\partial t} d \Omega+\int_{\Omega}\left(\frac{\partial \mathbf{f}}{\partial x}+\frac{\partial \mathbf{g}}{\partial y}\right) d \Omega=\int_{\Omega} \mathbf{s} d \Omega,
$$

where $\Omega$ denotes the area of a cell. Applying the divergence theorem and replacing the boundary integral with a sum over all edges, Eq. (4) becomes

$$
\int_{\Omega} \frac{\partial \mathbf{q}}{\partial t} d \Omega+\sum_{k=1}^{m} \mathbf{F} \cdot \mathbf{n}_{k} l_{k}=\int_{\Omega} \mathbf{s} d \Omega
$$

81 herein $m$ is the number of edges, $l$ is the length of the edge, and $\mathbf{n}=\left(n_{x}, n_{y}\right)^{T}$, is the unit normal vector pointing in the outward normal direction of the boundary edge, $\mathbf{F} \cdot \mathbf{n}$ is the flux vector normal to the boundary and can be 
84

written as

$$
\mathbf{F} \cdot \mathbf{n}=\left(\mathbf{f n}_{\mathrm{x}}+\mathbf{g n}_{\mathrm{y}}\right)=\left[\begin{array}{c}
q_{x} n_{x}+q_{y} n_{y} \\
\left(u q_{x}+g h^{2} / 2\right) n_{x}+v q_{y} n_{y} \\
u q_{x} n_{x}+\left(v q_{y}+g h^{2} / 2\right) n_{y}
\end{array}\right]
$$

The value of $\mathbf{q}$ in cell $i$ is updated using the two-stage explicit Runge-

Kutta scheme $[10,11,12]$, where the value at the next time level in cell $i$, $\mathbf{q}_{i}^{n+1}$, is updated by

$$
\mathbf{q}_{i}^{n+1}=\frac{1}{2}\left\{\mathbf{q}_{i}^{n}+\kappa\left[\kappa\left(\mathbf{q}_{i}^{n}\right)\right]\right\},
$$

with

$$
\kappa\left(\mathbf{q}_{i}^{n}\right)=\mathbf{q}_{i}^{n}+\frac{\Delta t}{\Omega}\left[\int_{\Omega} \mathbf{s} d \Omega-\sum_{k=1}^{m} \mathbf{F}\left(\mathbf{q}_{i}^{n}\right)_{k} \cdot \mathbf{n}_{k} l_{k}\right],
$$

where $\kappa$ is a function to represent the updating process to a new time level in the considered cell. $\Delta t$ is the time step. For this work, the CourantFriedrichs-Lewy condition is followed for maintaining the stability,

$$
\Delta t=\operatorname{CFL} \min \left(\frac{R_{1}}{\sqrt{u_{1}^{2}+v_{1}^{2}}+\sqrt{g h_{1}}}, \ldots, \frac{R_{n}}{\sqrt{u_{n}^{2}+v_{n}^{2}}+\sqrt{g h_{n}}}\right),
$$

where $R_{n}$ is the minimum distance from the cell center to the edge, CFL is the Courant-Friedrichs-Lewy number. For explicit time marching algorithms $\mathrm{CFL} \in(0,1]$. In this work, $\mathrm{CFL}=0.5$ is adopted.

\section{Multislope MUSCL reconstruction methods}

The original Godunov's theorem used cell-averaged values for calculating the flux and slope source terms. This is first order accurate. In order to get higher accuracy, a linear MUSCL reconstruction is usually used to obtain a second-order accurate scheme. Different ways for calculating slopes lead 
to different types of MUSCL reconstructions that give different performance (cf., e.g., $[13,14,15,12,16,17,18])$. In the multislope method, slopes are calculated towards each edge individually. As shown in Fig. 1, reconstructed values along the conjuncted edge are represented by $q_{M}^{l}$ and $q_{M}^{r}$. $M$ is the middle point of the edge, $N_{1-3}$ are the vertices of the left cell, cell averaged values of the left and right cells are represented by $q_{C}$ and $q_{D 1}$, respectively. Based on the work in [5], $q_{M}^{l}$ can be extrapolated from the cell centroid based on a one-dimensional multislope MUSCL by

$$
q_{M}^{l}=q_{C}+|\overrightarrow{C M}| \Psi\left(\nabla q_{N_{3} C}, \nabla q_{C M}\right),
$$

where $\overrightarrow{C M}$ is the vector from cell centroid $C$ to edge middle point $M$, and $\nabla q_{N_{3} C}$ and $\nabla q_{C M}$ represent the gradients from $N_{3}$ to cell center and from the cell center to edge center, respectively. $\Psi$ is the limiting function for restricting the reconstruction scheme to satisfy the total variation diminishing condition. The modified Van Albada's limiter with two arguments $a$ and $b$ from [19] is adopted in this work,

$$
\Psi(a, b)= \begin{cases}\frac{\left(a^{2}+e\right) b+\left(b^{2}+e\right) a}{a^{2}+b^{2}+2 e} & \text { if } a b>0 \\ 0 & \text { if } a b \leq 0\end{cases}
$$

Here, $e=10^{-12}$ used to avoid division by zero.

\subsection{Vector manipulation methods}

The two-dimensional multislope MUSCL schemes can be thought as a one-dimensional reconstruction process along the median line linking the cell center and the edge middle point, with the focus on the method for calculating the upwind and downwind slopes of the cell center along the median 
line. In the aforementioned literatures, slopes constructed by extrapolating the upwind value along the cell centrelines [4, 9, 3], and the approximated cell averaged method [20] have been studied. Owing to the accuracy and the unphysical reconstruction point location at the edge, the multislope method still needs to be further investigated.

Buffard and Clain [2] proposed the vector manipulation methods, where upwind and downwind slopes can be calculated without the gradient calculation for the cells and the interpolation for the upwind points. Hou et al. [5] simplified the original scheme to make the vector manipulation method more straightforward to implement and enhance the robustness and accuracy.

As mentioned in [8], the main idea of the vector manipulation method is to reconstruct the slopes from the cell centres to the slopes along the line passing through the cell center and the edge middle point. It has been shown that the methods from [2] and [5] include too much information from the considered cells and may lead to a wrong reconstruction value along the edge center. An improved vector manipulation method introduced in [8] overcomes this disadvantage.

The legends are shown in Fig. 1, and the dimensional unit vector can be calculated as,

$$
\begin{aligned}
& \overrightarrow{r_{k}}=\frac{\overrightarrow{C N_{k}}}{\left|\overrightarrow{C N_{k}}\right|} \\
& \overrightarrow{t_{k}}=\frac{\overrightarrow{C D_{k}}}{\left|\overrightarrow{C D_{k}}\right|},
\end{aligned}
$$

where $\overrightarrow{r_{k}}$ and $\overrightarrow{t_{k}}$ are the unit vectors from the considered cell center to the vertices and the neighboring cell centres, respectively. It can be easily shown 
that all the vector $\overrightarrow{r_{k}}$ shall pass by the corresponding edge center along the reverse direction.

The value slopes for the cell centres can be calculated along $\overrightarrow{t_{k}}$ directions,

$$
\nabla q_{k}=\frac{q_{D_{k}}-q_{C}}{\left|\overrightarrow{C D_{k}}\right|}
$$

The upwind and downwind slopes for the MUSCL reconstruction can be thought as the slopes along the reverse direction of $\overrightarrow{r_{k}}$ from the vertices to the cell center and cell center to the edge center. For instance, the reconstructed value $q_{M}^{l}$ needs the slope along $-\overrightarrow{r_{3}}$ and $\overrightarrow{r_{C M}}$, respectively. $M$ represents the middle of the edge.

In order to get the right information for the reconstruction, the unit vector $\overrightarrow{r_{k}}$ is represented by the surrounding unit vector $\overrightarrow{t_{k}}$, with the consideration of geometric relationship obtained as

$$
\begin{aligned}
& -\overrightarrow{r_{3}}=\alpha_{1} \overrightarrow{t_{2}}+\alpha_{2} \overrightarrow{t_{3}} \\
& \overrightarrow{r_{C M}}=\beta_{1} \overrightarrow{t_{1}}+\beta_{2} \overrightarrow{t_{2}} .
\end{aligned}
$$

Wherein, the coefficients $\alpha_{1,2}$ and $\beta_{1,2}$ can be solved by a set of linear equations. So that the upwind and downwind slopes can be computed as

$$
\begin{aligned}
& \nabla q_{N C}=\alpha_{1} \nabla q_{2}+\alpha_{2} \nabla q_{3} \\
& \nabla q_{C M}=\beta_{1} \nabla q_{1}+\beta_{2} \nabla q_{2} .
\end{aligned}
$$

In the work of Hou et al. [5], $\nabla q_{N C}$ and $\nabla q_{C M}$ are directly used as the upwind and downwind slopes for the MUSCL reconstruction. An additional step is added for obtaining more downwind information. From the geometric relationship, it can be concluded that

$$
\overrightarrow{r_{C M_{k}}}=\frac{\left|\overrightarrow{C D_{k}}\right|}{\left|\overrightarrow{C M_{k}}\right|} \overrightarrow{r_{C D_{k}}}+\frac{\left|\overrightarrow{D_{k} M_{k}}\right|}{\left|\overrightarrow{C M_{k}}\right|} \overrightarrow{r_{D M_{k}}}
$$


151

and then, Eq. 18 can be derived as

$$
\nabla q_{C M_{k}}=\frac{\left|\overrightarrow{C D_{k}}\right|}{\left|\overrightarrow{C M_{k}}\right|} \nabla q_{C D_{k}}+\frac{\left|\overrightarrow{D_{k} M_{k}}\right|}{\left|\overrightarrow{C M_{k}}\right|} \nabla q_{D_{k} M_{k}}
$$

Here, $k$ is the local index of the considered cell. This treatment has been approved to give more physical reconstructed value and obtain good accuracy in $[8]$.

\subsection{Improved vector manipulation method}

As discussed in the previous section, including solely upwind information decreases the stability of the scheme. The improved vector manipulation scheme obtains more information from downwind direction. However, the slope from cell centres to the edges centres $\overrightarrow{r_{C M_{k}}}$ needs to be calculated from the location relationships with the cell centres vectors $\overrightarrow{t_{k}}$. Additional computational steps are needed to decide $\overrightarrow{r_{C M_{k}}}$ located in which two cell centres vectors $\overrightarrow{t_{k}}$ before the calculation of Eq. 16, and the slope calculation is highly influenced by the geometric distribution rather than the physical values in VMM scheme. Therefore, an improved scheme is suggested here to overcome the disadvantage of the previous schemes.

As shown in Fig. 2, all the vectors from the cell centers to the vertices 
can be calculated as

$$
\begin{aligned}
& \overrightarrow{r_{1}}=\beta_{11} \overrightarrow{t_{1}}+\beta_{12} \overrightarrow{t_{2}} \\
& \overrightarrow{r_{2}}=\beta_{21} \overrightarrow{t_{1}}+\beta_{22} \overrightarrow{t_{3}} \\
& \overrightarrow{r_{3}}=\beta_{31} \overrightarrow{t_{2}}+\beta_{32} \overrightarrow{t_{3}} \\
& \overrightarrow{r_{4}}=\beta_{41} \overrightarrow{t_{4}}+\beta_{42} \overrightarrow{t_{5}} \\
& \overrightarrow{r_{5}}=\beta_{51} \overrightarrow{t_{4}}+\beta_{52} \overrightarrow{t_{6}} \\
& \overrightarrow{r_{6}}=\beta_{61} \overrightarrow{t_{5}}+\beta_{62} \overrightarrow{t_{6}}
\end{aligned}
$$

166

considering the unit vectors $r_{k}$, Eq. 27 can be written into

$$
\overrightarrow{r_{D M}}=\frac{\left|\overrightarrow{D N_{1}}\right|}{2|\overrightarrow{D M}|} \overrightarrow{r_{4}}+\frac{\left|\overrightarrow{D N_{2}}\right|}{2|\overrightarrow{D M}|} \overrightarrow{r_{5}},
$$

so that, Eq. 16 can be changed to

$$
\begin{aligned}
\overrightarrow{r_{C M}} & =\frac{|\overrightarrow{C D}|}{|\overrightarrow{C M}|} \overrightarrow{t_{1}}+\frac{|\overrightarrow{D M}|}{|\overrightarrow{C M}|}\left(\frac{\left|\overrightarrow{D N_{1}}\right|}{2|\overrightarrow{D M}|} \overrightarrow{r_{4}}+\frac{\left|\overrightarrow{D N_{2}}\right|}{2|\overrightarrow{D M}|} \overrightarrow{r_{5}}\right) \\
& =\frac{|\overrightarrow{C D}|}{|\overrightarrow{C M}|} \overrightarrow{t_{1}}+\frac{\left|\overrightarrow{D N_{1}}\right|}{2|\overrightarrow{C M}|} \overrightarrow{r_{4}}+\frac{\left|\overrightarrow{D N_{2}}\right|}{2|\overrightarrow{C M}|} \overrightarrow{r_{5}} \\
& =\frac{|\overrightarrow{C D}|}{|\overrightarrow{C M}|} \overrightarrow{t_{1}}+\frac{\left|\overrightarrow{D N_{1}}\right|}{2|\overrightarrow{C M}|}\left(\beta_{41} \overrightarrow{t_{4}}+\beta_{42} \overrightarrow{t_{5}}\right)+\frac{\left|\overrightarrow{D N_{2}}\right|}{2|\overrightarrow{C M}|}\left(\beta_{51} \overrightarrow{t_{4}}+\beta_{52} \overrightarrow{t_{6}}\right),
\end{aligned}
$$

the slope of cell centres are introduced, and then the downwind slope can be computed as

$$
\begin{aligned}
\nabla q_{C M} & =\frac{|\overrightarrow{C D}|}{|\overrightarrow{C M}|} \nabla q_{C D}+\frac{\left|\overrightarrow{D N_{1}}\right|}{2|\overrightarrow{C M}|}\left(\beta_{41} \nabla q_{D C}+\beta_{42} \nabla q_{D G}\right) \\
& +\frac{\left|\overrightarrow{D N_{2}}\right|}{2|\overrightarrow{C M}|}\left(\beta_{51} \nabla q_{D C}+\beta_{52} \nabla q_{D E}\right) .
\end{aligned}
$$


A local extrema violates the monotonicity principle [21]. The maximum principle states that the extrapolated value along the edge midpoints of the cell nodes can not be beyond the range of the local maximum and minimum value. It is originally proposed in [22] to avoid over- and undershooting when reconstructing the slopes for multi-dimensional problems on unstructured grids. For the one-dimensional problem, the maximum principle can be used as:

$$
\min \left(q_{l}, q_{r}\right) \leq q_{M}^{l}, q_{M}^{r} \leq \max \left(q_{l}, q_{r}\right) .
$$

For the multi-dimensional problems, the reconstruction processes should try to include more multi-dimensional flow physics [23]. The proposed MUSCL reconstruction here includes 6 cells in the computational stencils. As shown in Fig. 3, the schemes from Buffard and Clain [2] and Hou et al. [5] are based on 4 cells, while the improved vector manipulation method from Zhao et al. [8] is based on 5 cells. The vector manipulation methods satisfy the maximum principle. It is hard to say whether more cells will lead to higher accuracy, but the more information is included, the maximum principle will be extended to a bigger range for the stability conditions, the less sensitive to local mesh distribution and faithfully represents multi-dimensional flow physics [23]. However, we shall note that the benefit of adding more cells to the stencil can be expected to diminish after a certain number.

The aim of MUSCL reconstruction is to give values at the left and right cell interface that can be used to construct a Riemann problem and calculate the slope source term. The solution of the Riemann problem then yields the numerical flux in Eq. (6) [16] and the slope source will be added into the fluxes across the edge. In this work, a Harten, Lax and van Leer Riemann 
solver with the contact wave restored (HLLC) [24] is used. The positivity preserving hydrostatic reconstruction by [25] is used to maintain non-negative water depth and correct reconstruction of the Riemann states, and the Cproperty preserving divergence form of the bed slope source term proposed by Hou et al. [26] is used; the source term treatment does not influence the well-balanced property of the MUSCL schemes.

For the friction source term, the most straightforward technique is explicit in time. However, this approach yields numerical instabilities unless the time step size $\Delta t$ satisfies [27]:

$$
-1 \leq 1+\frac{\mathbf{s}\left(\mathbf{q}_{i}^{n+1, x}\right)}{\mathbf{q}_{i}^{n+1, x}} \Delta t \leq 1,
$$

where $\mathbf{q}_{i}^{n+1, x}$ is the solution after adding the fluxes terms, and the time step has to be calculated using

$$
\begin{aligned}
\Delta t_{\mathbf{s}} & =\underset{i=1, \ldots, N}{\operatorname{Min}}\left[-2 \frac{\mathbf{q}_{i}^{n+1, x}}{\mathbf{s}\left(\mathbf{q}_{i}^{n+1, x}\right)}\right] \\
\Delta t & =\operatorname{Min}\left(\Delta t_{c}, \Delta t_{\mathbf{s}}\right),
\end{aligned}
$$

where $\Delta t, \Delta t_{\mathrm{s}}$ and $\Delta t_{c}$ are time steps for the system, source term part and conservation part, respectively. Depending on the source term, this might result in a severe degradation of the time step size.

To overcome this limitation, in literature, e.g. [26, 12], the splitting pointimplicit method is adopted. This avoids the instability of the numerical scheme for very shallow water depths.

In splitting point implicit methods, conserved variables inside the cell are updated as

$$
\mathbf{q}^{n+1}=\mathbf{q}^{n}+\frac{1}{\mathbf{P I}}\left(-\frac{\Delta t}{A} \sum_{k} \mathbf{f}_{k}^{n} \cdot \mathbf{n}_{k} l_{k}+\Delta t \mathbf{s}^{n}\right)
$$


209

here, $n$ and $n+1$ represent the time levels and $\mathbf{P I}$ is a matrix equal to

$$
\mathbf{P I}=\mathbf{I}-\Delta t\left(\frac{\partial \mathbf{s}}{\partial \mathbf{q}}\right)^{n} .
$$

210

The momentum friction source terms are derived with respect to the unit discharge, except the slope source term that has been transformed into fluxes over the cell edges. Eq. 38 then yields

$$
\mathbf{P I}=\left[1-\Delta t\left(\partial s_{x} / \partial q_{x}\right)^{n}, 1-\Delta t\left(\partial s_{y} / \partial q_{y}\right)^{n}\right]^{T} .
$$

This gives

$$
\begin{aligned}
& \frac{\partial s_{x}}{\partial q_{x}}=-\frac{c_{f}}{h^{2}}\left(\hat{q}+\frac{q_{x}^{2}}{\hat{q}}\right), \\
& \frac{\partial s_{y}}{\partial q_{y}}=-\frac{c_{f}}{h^{2}}\left(\hat{q}+\frac{q_{y}^{2}}{\hat{q}}\right),
\end{aligned}
$$

where $\hat{q}=\sqrt{q_{x}^{2}+q_{y}^{2}}$ is the magnitude of the unit discharge vector.

In order to preserve the stability, the general treatment from [21] is adopted here, which locally switches the second order MUSCL scheme to first order in a cell when the flow condition satisfying:

$$
h_{M}^{L} \leq \min \left(\left|z_{b M}^{L}-z_{c}\right|, 0.25 h_{c}\right) \text { or } h_{c} \leq \epsilon_{w d}
$$

here, $h_{M}^{L}$ and $z_{M}^{L}$ represent the reconstructed water depth and bottom elevation, respectively, along the considered edge; $h_{c}$ and $z_{c}$ are the corresponding values at the cell center, $\epsilon_{w d}$ is the tolerance used to distinguish the wet and dry cells, which is set to $\epsilon_{w d}=10^{-6}$ in this study.

The procedures of MUSCL reconstruction methods for vector manipulation method (VMM) and the improved vector manipulation method (IVMM) are summarized in Table 1. 


\section{Numerical Tests}

Five computational test cases published in the literature are presented here for verifying the MUSCL reconstruction methods. The performance of MUSCL reconstruction methods will be evaluated in terms of accuracy and efficiency. Two types of meshes, namely the diagonal mesh and the Delaunay mesh, are considered in evaluating each MUSCL reconstruction, as seen in Fig. 4.

The first test case considers a Riemann problem from Toro [28] as a benchmark to verify the stability and the efficiency of the MUSCL schemes. Moving shorelines in a two-dimensional frictional parabolic bowl is chosen as the second test case, where the proposed MUSCL schemes are verified for the accuracy and the capability to deal with wet and dry interface. Meanwhile, the performance of the friction source term treatment, and the grid convergence performance are investigated based on this test. The third and the forth examples are the MUSCL schemes are tested against the dam-break in a $45^{\circ}$ channel and a two-dimensional dam-break flow against an isolated obstacle for evaluating how the MUSCL schemes perform on complex geometry for shock wave capturing. The final test cases are the near real-world application for the Malpasset dam-break. The accuracy is reflected by the $L_{1}$-error which can be calculated as

$$
L_{1}=\frac{\sum_{1}^{n}\left|q_{i}-q_{i, \mathrm{ref}}\right| A_{i}}{\sum_{1}^{n} A_{i}},
$$

which $q_{i}$ and $q_{i, \text { ref }}$ are the numerical solution and the reference solution in cell $i$, respectively. A characteristic length $\Delta x=\sqrt{A / N}$ is used here for the resolution of the meshes, $A$ and $N$ are the total area and the number of cells. 


\subsection{Dam break problems}

Two challenging problems proposed by Toro [28] are used here for examining the capability to resolve the linear and non-linear waves on unstructured grids. A frictionless rectangular channel with $[0,50] \times[0,0.25] \mathrm{m}$ is discretized into 12032 Delaunay triangular meshes. Initial conditions of the test cases are summarized in Tab. 2, where $h_{L}, h_{R}, u_{L}$ and $u_{R}$ donates the initial water depth and the velocity in the left and right hand side of the discontinuity, $x_{0}$ is the location of the discontinuity, $t_{\text {out }}$ is the output time.

Different initial conditions lead to different results after a short period, which the configuration $a$ leads to a result with the left wave as a rarefaction wave transport to the left and the right wave as a shock transport to the right. Configuration $b$ generate a two rarefaction wave transport to a opposite direction, in the middle of the computational domain, a very shallow water depth keeps a constant value. The results are compared considering the water elevation $h$ and hydraulic head calculated via $e=h+\frac{U^{2}}{2 g}$. The exact solution (-), numerical solutions from $\operatorname{VMM}(-*-)$ and $\operatorname{IVMM}(-\circ-)$ are shown in Fig. 5, it can be observed that the numerical results are quite coincide with the exact solution from Toro [28], there is a little diffusion at the front of the waves, the VMM and IVMM provide the same quality results. The comparisons of the computational efficiency are based on the averaged computational time for a single step $\Delta t$, which is calculated by $\Delta t=t_{\text {total }} / n$, where $t_{\text {total }}$ is the total computational time and $n$ is the number of time steps for the calculation. Each $\Delta t$ in VMM is bigger than in IVMM for 3.6\%, $2.9 \%$ in configuration $a$ and $b$, respectively. This means that IVMM can obtain a better efficiency than VMM scheme. The result is as expected in the previous 
section, the additional step used for searching the vectors $t$ will increase the computational time and decrease the computational efficiency.

\subsection{Moving shorelines in a two-dimensional frictional parabolic bowl}

The analytical solution of the moving shorelines in a two-dimensional frictional parabolic bowl was developed by Sampson et al. [29], it will be used to validate the proposed model for the MUSCL reconstruction and frictional treatment here. The bed topography is described as,

$$
z(x, y)=h_{0}\left[\left(x-x_{0}\right)^{2}+\left(y-y_{0}\right)^{2}\right] / a^{2} .
$$

Here, $z(x, y)$ represent the bottom elevation of the $(x, y)$ point, $x_{0}, y_{0}$ is the coordinate of the geometry center. $h_{0}$ is the initial water depth at the parabola center, and $a$ is a constant value. $\tau$ is the bed frictional parameter, and $c_{f}=h \tau / \sqrt{u^{2}+v^{2}}$. The peak amplitude parameter $p=\sqrt{8 g h_{0}} / a$, if $\tau<p$, the analytical solution for the water level is given by

$$
\begin{aligned}
\eta(x, y, t) & =h_{0}-\frac{B^{2} e^{-\tau t}}{2 g}-\frac{B e^{-\tau t / 2}}{g}\left\{\left[\frac{\tau \sin (s t)}{2}+s \cos (s t)\right]\left(x-x_{0}\right)\right. \\
& \left.+\left[\frac{\tau \cos (s t)}{2}-s \sin (s t)\right]\left(y-y_{0}\right)\right\}
\end{aligned}
$$

and the analytical solution for the velocities are

$$
\begin{aligned}
& u(t)=B e^{-\tau t / 2} \sin (s t) \\
& v(t)=B e^{-\tau t / 2} \cos (s t),
\end{aligned}
$$

where $B$ is a constant as an initial value of $v(0)$, and $s=\sqrt{p^{2}-\tau^{2}} / 2$, in which $p=\sqrt{8 g h_{0}} / a$.

A square computational domain of $8000 \times 8000 \mathrm{~m}$ with the center at $(0.0,0.0)$ is chosen as the study area. The parameters are set to $h_{0}=10 \mathrm{~m}$, 
$a=3000, B=5 \mathrm{~m} / \mathrm{s}$ and $\tau=0.002 \mathrm{~s}^{-1}$. The computational domain is discretized with two types of meshes with 5 refinement levels for the mesh convergence. The boundaries are all set to closed boundaries, and the simulation time runs until $t=6000 \mathrm{~s}$, which is almost 4 periods. The initial condition can be obtained from Eqs. 45 - 47.

The contours plot at $t=1500 \mathrm{~s}$, which is almost 1.1 period after the simulation begin can be seen in Fig. 6. The difference between VMM and IVMM scheme results is quite small, both of the schemes can capture the water depth quite well at diagonal grid and Delaunay grid at the first mesh level. The cut section plot along the diagonal line of the computational domain at $t=500 \mathrm{~s}$ and $t=1500 \mathrm{~s}$ are shown in Fig. 7 and Fig. 8 for the Delaunay and diagonal grids, respectively. In order to show the accuracy of the MUSCL reconstruction, the results at the first mesh level (finest mesh) and the fifth mesh level (coarsest mesh) are chosen for the comparison. The water level $(w l)$ and the discharge along $x$ - direction $\left(q_{x}\right)$ and $y$ - direction $\left(q_{y}\right)$ are all captured well with the analytical solution at the finest mesh; for the coarsest mesh, water levels $w l$ are captured well for both grids types, but for the discharges, it can be observed that the results of diagonal grids agree worse than that of Delaunay grids, especially for $q_{x}$, and the result from IVMM is slightly better than the VMM scheme.

A mesh convergence study for this test case at $t=1500 \mathrm{~s}$ is presented in Fig. 9. The $L_{1}$ errors for $h$ and $q_{y}$ are plotted in the figures for the different mesh level (represented by the characteristic length $\Delta x$ shown in Tab. 3). The results from VMM and IVMM are represented by the $\circ-\circ$ and $\triangleright-\triangleright$, respectively. It can be seen that the order of VMM and IVMM are all 
slightly lower than the slope 2 (solid lines). This is because of the wet and dry interfaces, where the order of the scheme will switch to first order, which decreases the overall order of accuracy. The VMM and IVMM schemes are nearly parallel with the increasing of the mesh level, but the error values for the IVMM scheme is almost always smaller than the corresponding error for the VMM scheme, which can be thought the order of the IVMM scheme and VMM scheme is similar but the accuracy of the IVMM scheme is better. As the characteristic length $\Delta x$ is different for the Delaunay and diagonal grids, the errors for the diagonal grids is a little bit higher than for the Delaunay grids. It was already shown the diagonal grids will significantly influence the results for the MUSCL reconstructions in [8], it can be observed here that the $q_{y}$ for the VMM scheme leads to higher error compared to the results from IVMM scheme, which means the IVMM is less influenced from the grid type.

The relative time of VMM against IVMM scheme is shown in Fig. 10. It can be clearly observed that IVMM provides a relatively better efficiency than VMM scheme, and with the increasing of the mesh number, the advantage becomes bigger. The unstructured grids in this work are mainly focused on the triangle mesh, where the vector structure in the single cell is still simple, but for a more complex mesh, the additional calculation will increase, which will decrease the computational efficiency even more.

\subsection{Dam-break in a channel with $45^{\circ}$ bend}

To assess the performance of the MUSCL reconstructions for the dambreak induced waves in non-straight channels, a test case from EU CADAM [30] is chosen as the benchmark, which was also considered in $[12,31,32]$ for 
verifying the capability of their model for dam-break simulation. The set up and the computational grid used for the simulation of the experiment facility can be seen in Fig. 11. The reservoir with the size $2.39 \times 2.44 \mathrm{~m}^{2}$ is located at the left side of the experiment, the northwest of the reservoir is set to be the origin position of the geometry, a $0.495 \mathrm{~m}$ wide channel with a $45^{\circ}$ bending corner is connected with the reservoir and with a free outlet for the end of the channel. The water depth for the reservoir and the channel are $0.58 \mathrm{~m}$ and $0 \mathrm{~m}$, respectively. A $0.33 \mathrm{~m}$ high topography step is located between the reservoir and the channel. The Manning number is suggested equal to $0.012 \mathrm{~s} / \mathrm{m}^{1 / 3}$ after the preliminary numerical tests. The computational domain is discretized into 13038 Delaunay triangles-based meshes.

The simulation results from VMM and IVMM scheme are compared with the measurement data for three gauges located as shown in Tab. 4. As shown in Fig. 12, after $40 \mathrm{~s}$, the water elevation is quite well predicted by the numerical results, the only overestimated water elevation is after $5 \mathrm{~s}$ at G6 and underestimated after 20s at G9, which may come from the three dimensional effects after the $45^{\circ}$ bend. It also can be observed that the difference between the IVMM and VMM scheme is quite small, they all provide promising result, but again, the IVMM is about $4.1 \%$ faster than the VMM scheme, which indicates that the proposed scheme is sufficient for simulating the dam-break flow over dry bed even discontinuity.

\subsection{Two-dimensional dam-break flow against an isolated obstacle}

A physical experiment is set up for two-dimensional dam-break flow against an isolated obstacle constructed by Soares-Frazão and Zech [33]. It is chosen for testing the capability of the MUSCL reconstructions work on asymmet- 
ric geometry, the water elevation and the velocity will be checked for both numerical schemes. The sketch of the experiment is shown in Fig. 13, with a trapezoidal bottom for the up and downstream channel and the cut sections can be found in Fig. 13, all the boundaries are closed except for the channel outlet. The initial water level for the reservoir and down stream of the dam are $0.4 \mathrm{~m}$ and $0.02 \mathrm{~m}$, respectively. The dam-break is simulated by removing the gate in a sudden period. The velocities and water levels are measured in the different gauges located in the positions shown in Tab. 5, and the coordinate origin is set at the center of the gate.

The computational domain is discretized into 27831 triangle cells, relatively coarse mesh in the reservoir and a higher resolution for the downstream of the dam. The velocity field is set to be still for the beginning of the simulation. Numerical test will run for $30 \mathrm{~s}$ and the Manning coefficient is chosen $n=0.01 \mathrm{~s} / \mathrm{m}^{1 / 3}$ by following [33].

After $30 \mathrm{~s}$, the simulation results from VMM and IVMM compared with the measurement data are shown in Fig. 15, the water elevation is shown in the left column. It can be observed that the measured data is fairly good predicted by the numerical results, both MUSCL reconstructions show good agreement, but IVMM shows a little bit better results at gauge G2, however, the VMM is slightly better at G1. However, the IVMM leads to more stable results. The middle column presents the velocity along the $x$ - direction, the measurement data agrees well with the numerical results except for the G1, this maybe caused by the strong three dimensional effects near the obstacle. The VMM shows a slightly faster wave front at G5, here VMM may give a better prediction because of the faster wave speed, but it is difficult to say 
which one is better. The right column shows the velocity along the $y$ - direction, it can be observed that the range of the velocity value is smaller than the measurement data, as the obstacle provides a three dimensional influence on the flow field, which is neglected by the shallow water model. However, the water level at G6 seen in Fig. 16, shows that the numerical results perfectly captured the measured data, which means that both of the schemes can capture the long wave well. Again, the single computational effort is compared, and the IVMM can save $9.51 \%$ computational time compared to the IVMM scheme.

\subsection{Malpasset dam-break}

The last example is chosen to be the Malpasset dam-break for test the capability of the numerical model for simulating the field scale case. The Malpasset dam is located on the Reyran River valley and the associated floodplain in southern France is shown as in Fig. 17 (a). The topography is provided by [34] and the computational domain is discretized into 28855 triangle cells as shown in Fig. 17 (b) and the boundaries are set to be solid walls except for the downstream boundaries near to the sea which is transmissive. The reservoir has a constant water level for $100 \mathrm{~m}$ above the sea level, and the downstream of the dam is set to be initially dry except for the sea. The Manning coefficient is set to $0.033 \mathrm{~s} / \mathrm{m}^{1 / 3}$, following $[35,5,26$, $34,36,12]$.

Laboratory studies were carried out by Electricité de France to measure the arrival time and the maximum water level at the gauge points $\mathrm{G}$ (614) and the police points $P$ (1-17), the measurement data is well matched with the field data, and will be used for validating the numerical schemes. 
Simulation runs until 3600s and the water depth floodplain simulated by IVMM scheme is shown as in Fig. 18.

After $3600 \mathrm{~s}$, the arriving time at the electrical transformers is compared in Fig. 19(a), which the IVMM scheme reaches a little faster than the VMM scheme, being closer to the measurement data. The summary of the maximum water level of the survey points is shown in Fig. 19(b), it can be observed that the simulated results from both MUSCL schemes show fairly well agreement with the measurement data. Small discrepancies happen at the experiment gauges for the arriving time of the water, this can be due to the limitation of the two-dimensional SWEs and certain complex flows with three-dimensional effects will also influence the measurement results. This simulated results also well match the results from the literature, e.g. $[5,26,12]$. However, in general, the simulated results provided by VMM and IVMM can well predict the field measurements, there is no negative water depth predicted, nor are non-physical velocities created by the proposed schemes. To the end, the computational efficiency is compared and the IVMM saves $10.5 \%$ computation time compared to the VMM scheme.

\section{Conclusions}

An improved vector manipulation of the multislope MUSCL method is proposed in this work to achieve high accuracy and efficiency for the twodimensional unstructured cell-centered finite volume modelling of shallow water flows. The proposed scheme is proven to be more straight-forward without including any additional step for judging the geometry relationships.

Five examples involving analytical solution, laboratory experiments and field- 
scale surveys are used for validating the proposed scheme, and all the results are compared with those of the original vector manipulation method from [8]. The results from the proposed MUSCL reconstruction are shown to produce satisfactory results without creating negative water depth and infinite velocity. The mesh convergence study shows that the new scheme is roughly second order accuracy. The computational cost is compared in each test case, the new IVMM scheme is shown to save about 4\%-10\% computational time compared to the VMM scheme, and the saving is more apparent with more computational grid points. To sum up, the new reconstruction method exhibits good performance for solving the SWEs on unstructured grids.

\section{Acknowledgements}

The authors are grateful to the China Scholarship Council and TU Berlin for the scholarships granted to J. Zhao.

[1] B. van Leer, Towards the ultimate conservative difference scheme. V. A second-order sequel to Godunov's method, Journal of Computational Physics 32 (1979) 101-136.

[2] T. Buffard, S. Clain, Monoslope and multislope MUSCL methods for unstructured meshes, Journal of Computational Physics 229 (2010) 37453776 .

[3] M. Darwish, F. Moukalled, TVD schemes for unstructured grids, International Journal of Heat and Mass Transfer 46 (2003) 599-611. 
[4] J. Hou, F. Simons, R. Hinkelmann, Improved total variation diminishing schemes for advection simulation on arbitrary grids, International Journal for Numerical Methods in Fluids 70 (2012) 359-382.

[5] J. Hou, Q. Liang, H. Zhang, R. Hinkelmann, Multislope MUSCL method applied to solve shallow water equations, Computers \& Mathematics with Applications m (2014).

[6] S. K. Godunov, A difference method for numerical calculation of discontinuous equations of hydrodynamics (in Russian), Matematicheskii Sbornik 47 (1959) 271-300.

[7] V. Venkatakrishnan, Convergence to Steady State Solutions of the Euler Equations on Unstructured Grids with Limiters, Journal of Computational Physics 118 (1995) 120-130.

[8] J. Zhao, I. Özgen, D. Liang, R. Hinkelmann, Improved multislope muscl reconstruction on unstructured grids for shallow water equations, International Journal for Numerical Methods in Fluids 0 (2018) 1-36.

[9] X. Li, H. Liao, An improved r-factor algorithm for TVD schemes, International Journal of Heat and Mass Transfer 51 (2008) 610-617.

[10] Q. Liang, A. G. Borthwick, Adaptive quadtree simulation of shallow flows with wetdry fronts over complex topography, Computers \& Fluids 38 (2009) 221-234.

[11] Q. Liang, F. Marche, Numerical resolution of well-balanced shallow water equations with complex source terms, Advances in Water Resources 32 (2009) 873-884. 
[12] J. Hou, F. Simons, M. Mahgoub, R. Hinkelmann, A robust well-balanced model on unstructured grids for shallow water flows with wetting and drying over complex topography, Computer Methods in Applied Mechanics and Engineering 257 (2013) 126-149.

[13] F. Simons, T. Busse, J. Hou, I. Özgen, R. Hinkelmann, A model for overland flow and associated processes within the Hydroinformatics Modelling System, Journal of Hydroinformatics (2014) 1-26.

[14] Q. Liang, F. Marche, Numerical resolution of well-balanced shallow water equations with complex source terms, Advances in Water Resources 32 (2009) 873-884.

[15] Q. Liang, Flood Simulation Using a Well-Balanced Shallow Flow Model, Journal of Hydraulic Engineering 136 (2010) 669-675.

[16] E. F. Toro, Riemann Solvers and Numerical Methods for Fluid Dynamics, Springer-Verlag, Berlin Heidelberg, 3 edition, 2009.

[17] T. Buffard, S. Clain, Monoslope and multislope MUSCL methods for unstructured meshes, Journal of Computational Physics 229 (2010) 37453776 .

[18] V. Guinot, C. Delenne, MUSCL schemes for the shallow water sensitivity equations with passive scalar transport, Computers and Fluids 59 (2012) $11-30$.

[19] A. Delis, I. Nikolos, A novel multidimensional solution reconstruction and edge-based limiting procedure for unstructured cell-centered finite 
volumes with application to shallow water dynamics, International Journal for Numerical Methods in Fluids 71 (2013) 584-633.

[20] J. Hou, F. Simons, R. Hinkelmann, A new TVD method for advection simulation on 2D unstructured grids, International Journal for Numerical Methods in Fluids 71 (2013) 1260-1281.

[21] J. Hou, Q. Liang, H. Zhang, R. Hinkelmann, An efficient unstructured muscl scheme for solving the 2d shallow water equations, Environmental Modelling \& Software 66 (2015) 131-152.

[22] X.-D. Liu, A maximum principle satisfying modification of triangle based adapative stencils for the solution of scalar hyperbolic conservation laws, SIAM journal on numerical analysis 30 (1993) 701-716.

[23] J. S. Park, S. H. Yoon, C. Kim, Multi-dimensional limiting process for hyperbolic conservation laws on unstructured grids, Journal of Computational Physics 229 (2010) 788-812.

[24] E. F. Toro, M. Spruce, W. Speares, Restoration of the contact surface in the HLL-Riemann solver, Shock Waves 4 (1994) 25-34.

[25] E. Audusse, F. Bouchut, M.-O. Bristeau, R. Klein, B. Perthame, A Fast and Stable Well-Balanced Scheme with Hydrostatic Reconstruction for Shallow Water Flows, SIAM Journal on Scientific Computing 25 (2004) 2050-2065.

[26] J. Hou, Q. Liang, F. Simons, R. Hinkelmann, A 2D well-balanced shallow flow model for unstructured grids with novel slope source term treatment, Advances in Water Resources 52 (2013) 107-131. 
[27] V. Guinot, Godunov-type Schemes: An introduction for engineers, Elsevier Science B.V., Amsterdam, The Netherlands, 1 edition, 2003.

[28] E. F. Toro, Shock-capturing Methods for Free-surface Shallow Flows, John Wiley \& Sons, New York/Chichester, 2001.

[29] J. Sampson, A. Easton, M. Singh, Moving boundary shallow water flow above parabolic bottom topography, Anziam Journal 47 (2006) 373-387.

[30] M. Morris, Cadam concerted action on dambreak modelling, Report SR $571(2000)$.

[31] H.-M. Kao, T.-J. Chang, Numerical modeling of dambreak-induced flood and inundation using smoothed particle hydrodynamics, Journal of hydrology 448 (2012) 232-244.

[32] J. G. Zhou, D. M. Causon, C. G. Mingham, D. M. Ingram, Numerical prediction of dam-break flows in general geometries with complex bed topography, Journal of hydraulic engineering 130 (2004) 332-340.

[33] S. Soares-Frazão, Y. Zech, Experimental study of dam-break flow against an isolated obstacle, Journal of Hydraulic Research 45 (2007) $27-36$.

[34] G. N., The Malpasset dam failure. An overview and test case definition, Proceedings of CADAM Zaragoza meeting (1999) 1-7.

[35] Y. Wang, Q. Liang, G. Kesserwani, J. W. Hall, A 2d shallow flow model for practical dam-break simulations, Journal of Hydraulic Research 49 (2011) 307-316. 
545 [36] A. Valiani, V. Caleffi, A. Zanni, Case study: Malpasset dam-break simulation using a two-dimensional finite volume method, Journal of $547 \quad$ Hydraulic Engineering 128 (2002) 460-472. 
Table 1: Procedures of MUSCL schemes, choose Fig. 2 as legend

\begin{tabular}{|c|c|c|}
\hline Steps & $\begin{array}{l}\text { Vector Manipulation Method } \\
(\mathrm{VMM})\end{array}$ & $\begin{array}{l}\text { Improved Vector Manipulation } \\
\text { Method (IVMM) }\end{array}$ \\
\hline 1 & Compute $\overrightarrow{r_{k}}$ and $\overrightarrow{t_{k}}$ & Compute $\overrightarrow{r_{k}}$ and $\overrightarrow{t_{k}}$ \\
\hline 2 & $\begin{array}{l}\text { Solve Eqs. }(15 \& 16) \text { to get } \\
\text { the coefficients } \alpha_{1}, \alpha_{2}, \beta_{1} \text { and } \\
\beta_{2} \text {. }\end{array}$ & $\begin{array}{l}\text { Solve Eqs. (21-26) to get } \\
\beta_{1-6,1-2}\end{array}$ \\
\hline 3 & $\begin{array}{l}\text { Calculate } \nabla q_{C,(D, F, H)} \text { and } \\
\nabla q_{D,(C, E, G)} \text { from Eq. }(14)\end{array}$ & $\begin{array}{l}\text { Calculate } \nabla q_{C,(D, F, H)} \text { and } \\
\nabla q_{D,(C, E, G)} \text { from Eq. (14) }\end{array}$ \\
\hline 4 & $\begin{array}{l}\text { Evaluate } \nabla q_{N C} \text { and } \nabla q_{C M} \\
\text { from Eq. }(17 \& 18)\end{array}$ & $\begin{array}{l}\text { Evaluate } \nabla q_{N C} \text { and } \nabla q_{C M} \\
\text { from Eq. }(17 \& 32)\end{array}$ \\
\hline 5 & Update $\nabla q_{C M}$ with Eq. (20) & Update $\nabla q_{C M}$ with Eq. $(20)$ \\
\hline 6 & $\begin{array}{l}\text { Extrapolate the edge value by } \\
\text { following Eq. (10) }\end{array}$ & $\begin{array}{l}\text { Extrapolate the edge value by } \\
\text { following Eq. (10) }\end{array}$ \\
\hline
\end{tabular}

Table 2: Initial conditions for Toro's test problems.

\begin{tabular}{ccccccc}
\hline Test & $h_{L}(\mathrm{~m})$ & $u_{L}(\mathrm{~m} / \mathrm{s})$ & $h_{R}(\mathrm{~m})$ & $u_{R}(\mathrm{~m} / \mathrm{s})$ & $x_{0}(\mathrm{~m})$ & $t_{\text {out }}(\mathrm{s})$ \\
\hline $\mathrm{a}$ & 1.0 & 2.5 & 0.1 & 0.0 & 10.0 & 7.0 \\
$\mathrm{~b}$ & 1.0 & -5.0 & 1.0 & 5.0 & 25.0 & 2.5 \\
\hline
\end{tabular}


Table 3: Characteristic length $\Delta x(\mathrm{~m})$ used for the mesh convergence test

\begin{tabular}{lll}
\hline Mesh Level & Diagonal Mesh & Delaunay Mesh \\
\hline 1 & 35.355 & 30.708 \\
2 & 52.868 & 45.967 \\
3 & 70.711 & 61.612 \\
4 & 88.386 & 77.110 \\
5 & 104.752 & 91.630 \\
\hline
\end{tabular}

Table 4: Position of measurement gauges.

\begin{tabular}{lll}
\hline Gauge & $\mathrm{x}[\mathrm{m}]$ & $\mathrm{y}[\mathrm{m}]$ \\
\hline 4 & 5.7400 & 0.6925 \\
6 & 6.6488 & 0.7650 \\
9 & 8.1267 & 2.2428 \\
\hline
\end{tabular}


Table 5: Two-dimensional dam-break flow against an isolated obstacle: position of measurement gauges.

\begin{tabular}{ccc}
\hline Gauge & $\mathrm{x}(\mathrm{m})$ & $\mathrm{y}(\mathrm{m})$ \\
\hline G1 & 2.65 & 1.15 \\
G2 & 2.65 & -0.60 \\
G3 & 4.00 & 1.15 \\
G4 & 4.00 & -0.80 \\
G5 & 5.20 & 0.30 \\
G6 & -1.87 & 1.10 \\
\hline
\end{tabular}

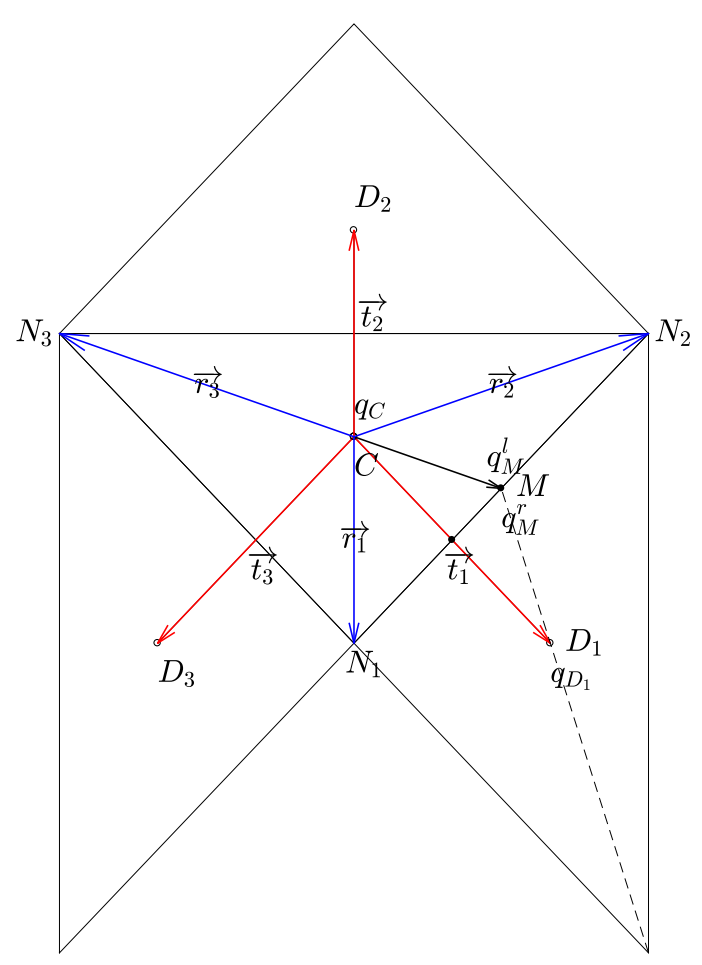

Figure 1: Definition of the considered cell and the neighbor cells. 


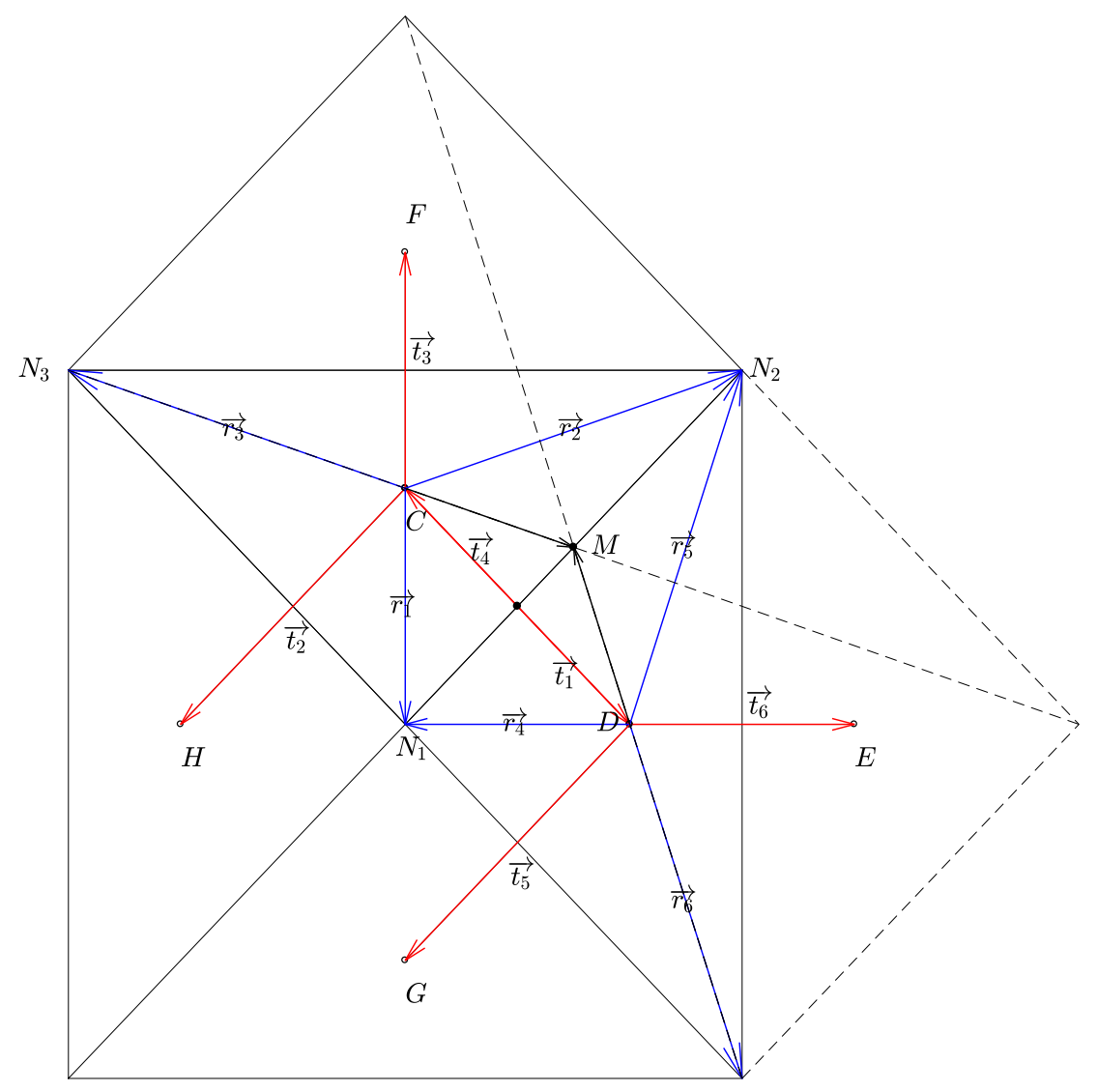

Figure 2: Stencils for new vector manipulation method. 


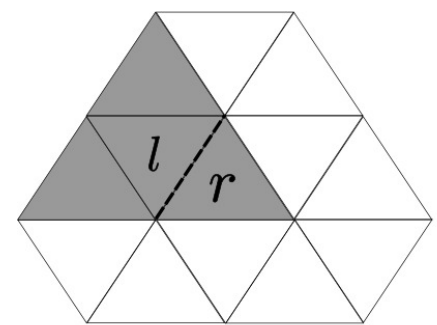

(a)

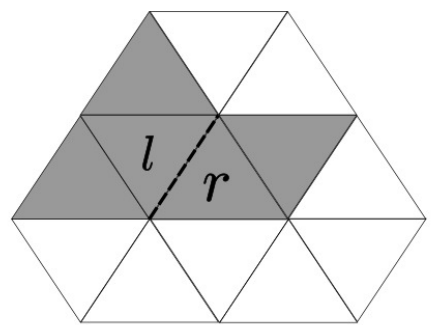

(b)

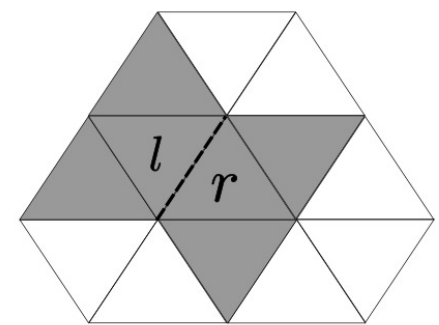

(c)

Figure 3: Comparison of stencils involved in limiting and the maximum principle. Shaded region is the stencil for the maximum principle, and the dotted line is the edge for limiting. (a) Limiter from Buffard and Clain [2] and Hou et al. [5], (b) limiter from Zhao et al. [8] and (c) new limiter in this work.
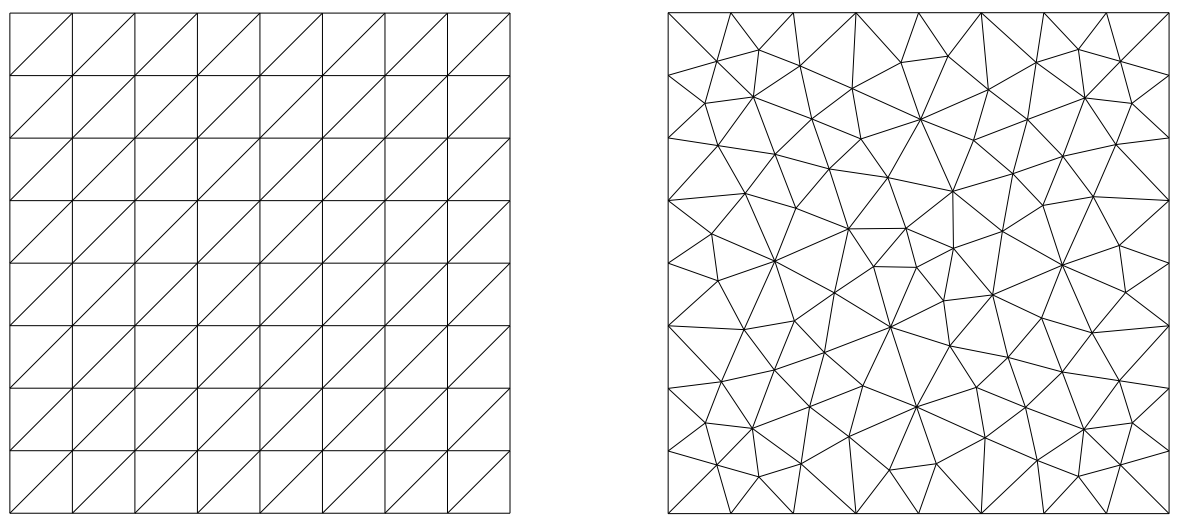

Figure 4: The two types of mesh employed to evaluate the accuracy and efficiency of the schemes: the diagonal mesh (left) and the Delaunay mesh (right) 

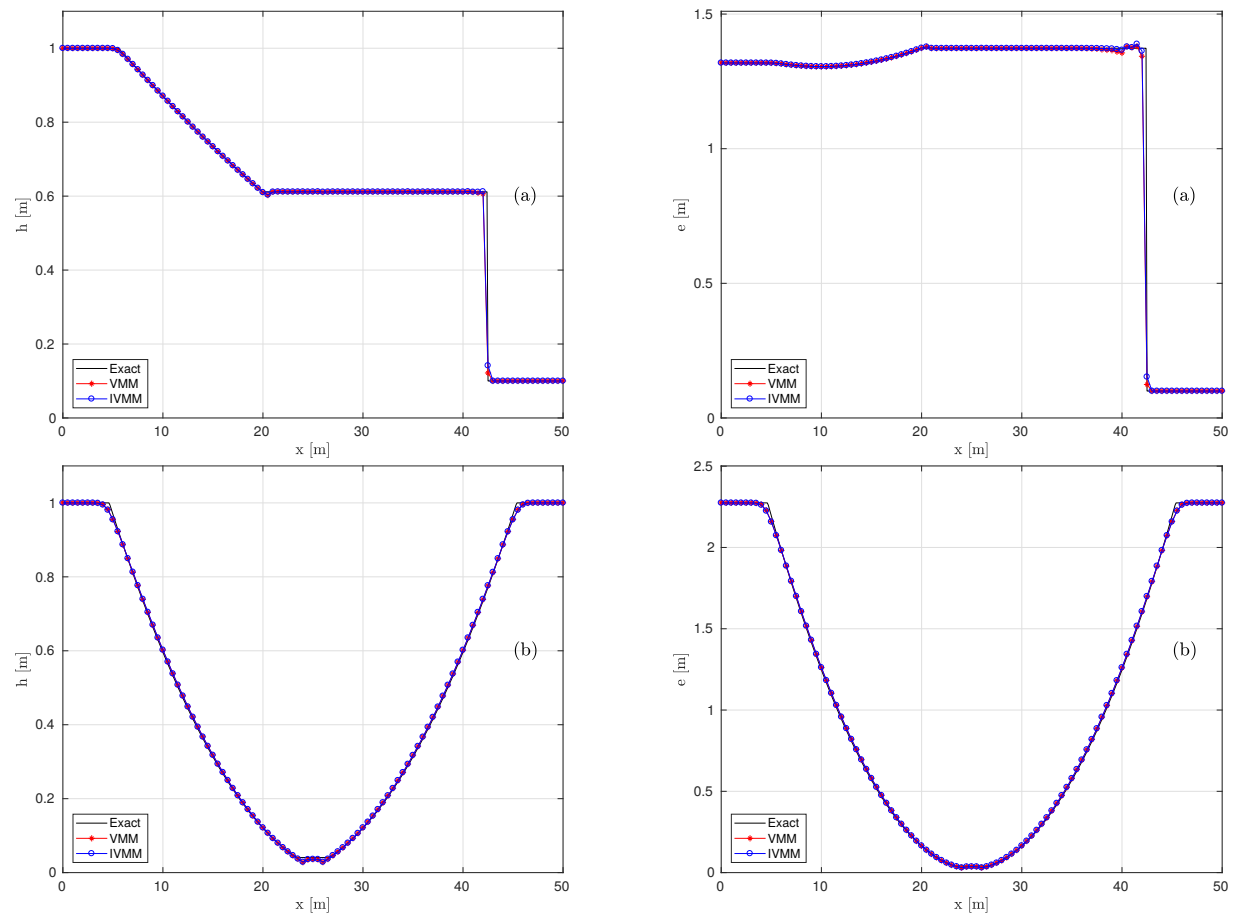

Figure 5: Comparison of numerical and exact solution for Toro's Riemann problems: left rarefaction wave and right shock wave: water elevation (a left), hydraulic head (a right); two rarefaction waves and nearly dry bed: water elevation (b left), hydraulic head (b right). 

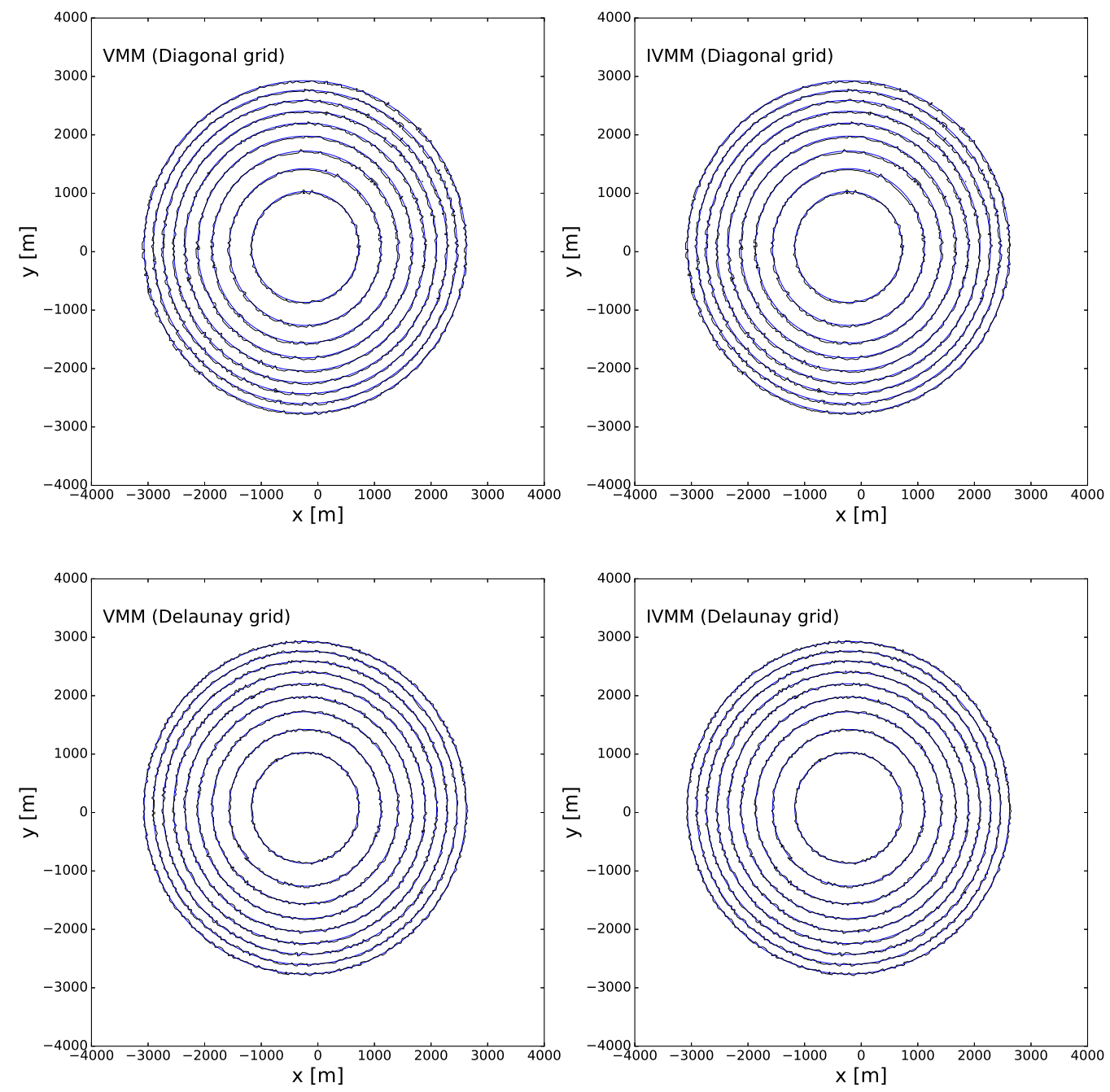

Figure 6: Moving shorelines in a two-dimensional frictional parabolic bowl: contours of water depth computed by VMM scheme (black lines located at the left side) and the result from IVMM scheme (black lines located at the right side) by using the diagonal grid (level 5 , for the upper part) and the Delaunay grid (level 5, for the lower part) compared with the analytical solution (blue lines) at $t=1500 \mathrm{~s}$. 

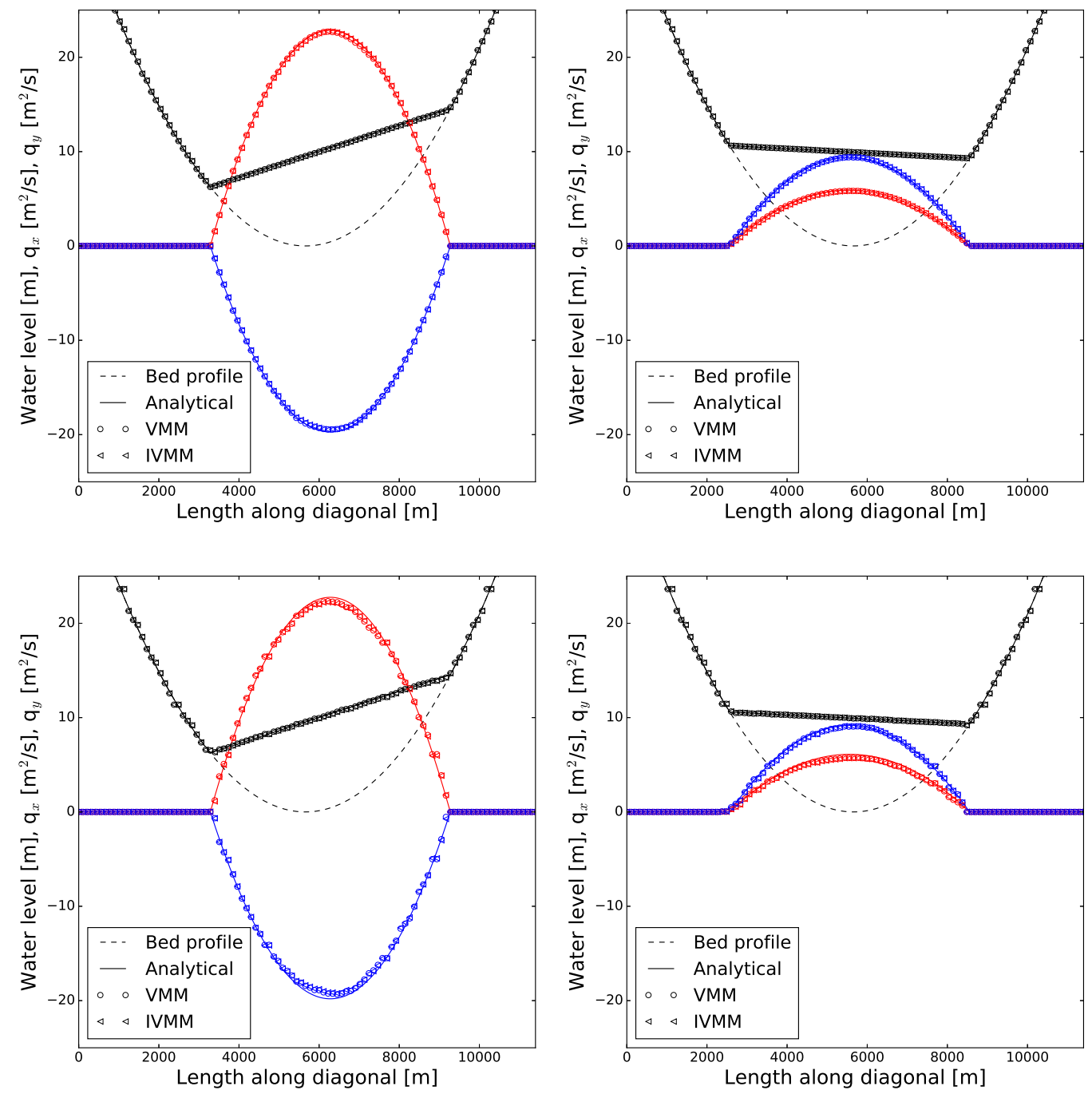

Figure 7: Moving shorelines in a two-dimensional frictional parabolic bowl: comparison between analytical solution (-) and the numerical solution (Delaunay mesh): $\operatorname{VMM}(\circ \circ)$, $\operatorname{IVMM}(\triangleleft \triangleleft)$ for the water level (black), $q_{x}$ (red), $q_{y}$ (blue) for $t=500 \mathrm{~s}$ (left) and $t=1500$ $\mathrm{s}$ (right) for the Delaunay grid in level 1 (upper) and level 5 (lower). 

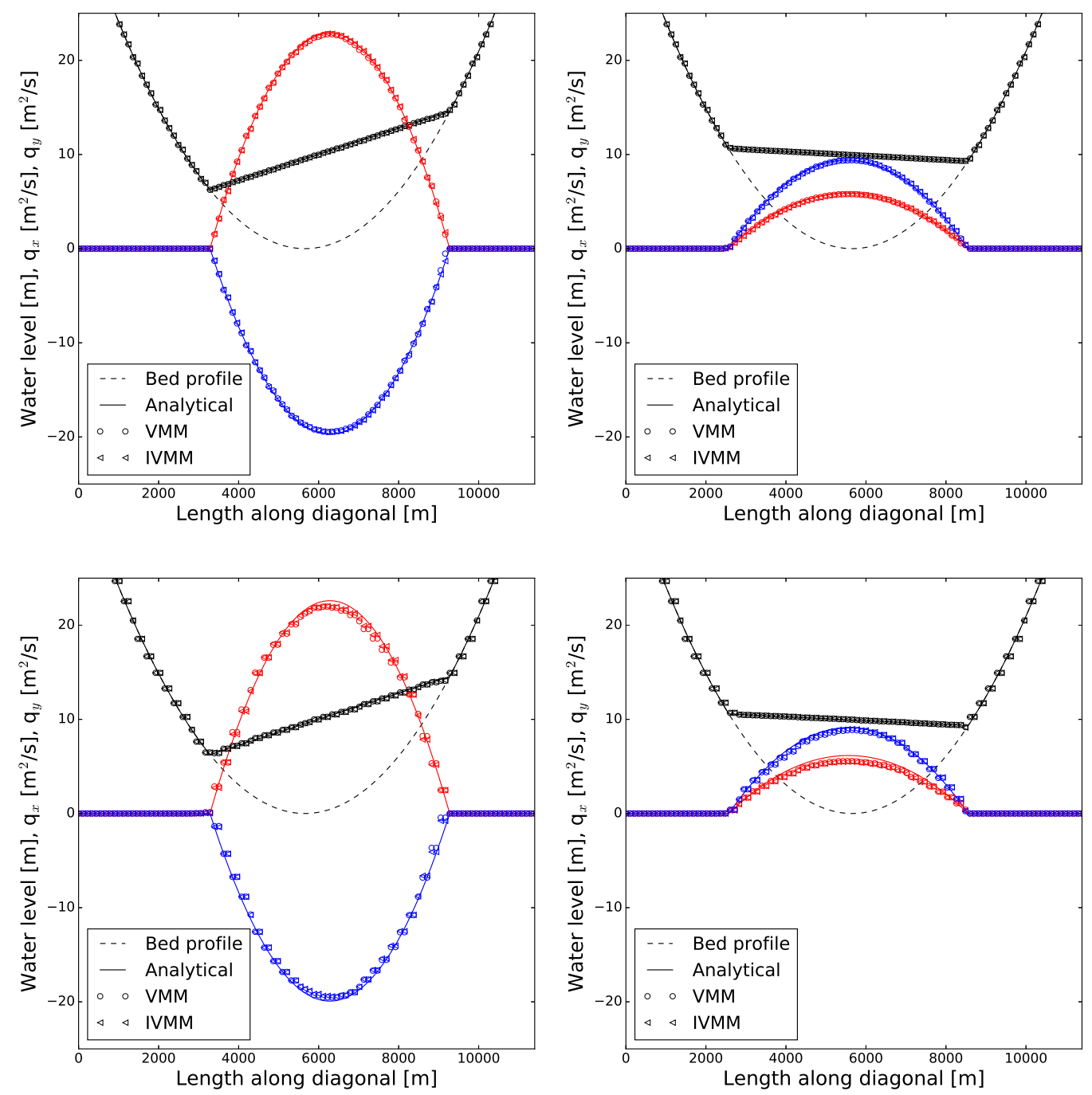

Figure 8: Moving shorelines in a two-dimensional frictional parabolic bowl: comparison between analytical solution (-) and the numerical solution (diagonal mesh): $\operatorname{VMM}(\circ \circ)$, $\operatorname{IVMM}(\triangleleft \triangleleft)$ for the water level (black), $q_{x}$ (red), $q_{y}$ (blue) for $t=500 \mathrm{~s}$ (left) and $t=1500$ $\mathrm{s}$ (right) for the diagonal grid in level 1 (upper) and level 5 (lower). 

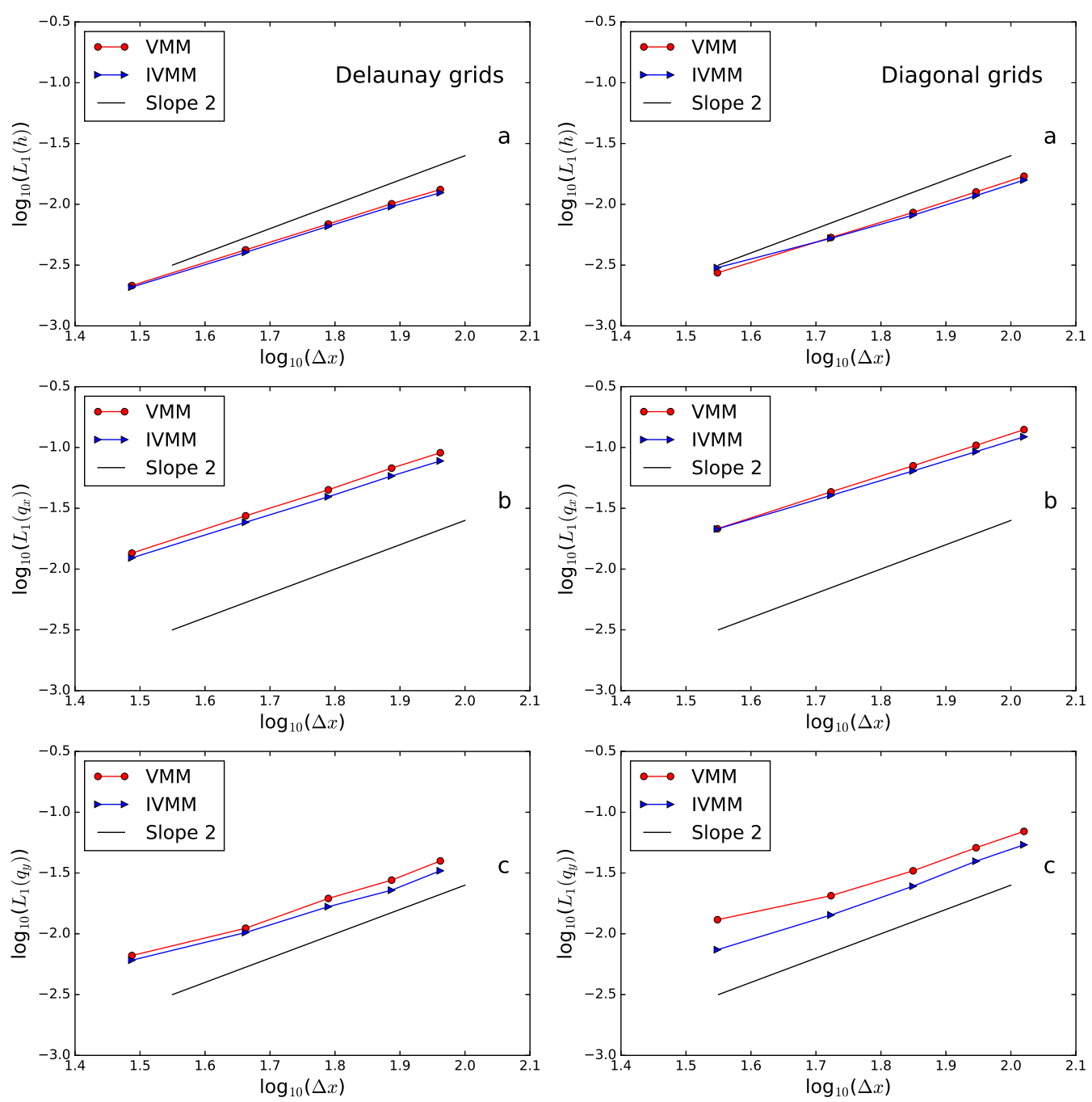

Figure 9: Moving shorelines in a two-dimensional frictional parabolic bowl: grid convergence study on Delaunay grids (left) and diagonal grids (right) for h (a), $q_{x}$ (b) and $q_{y}$ (c). 

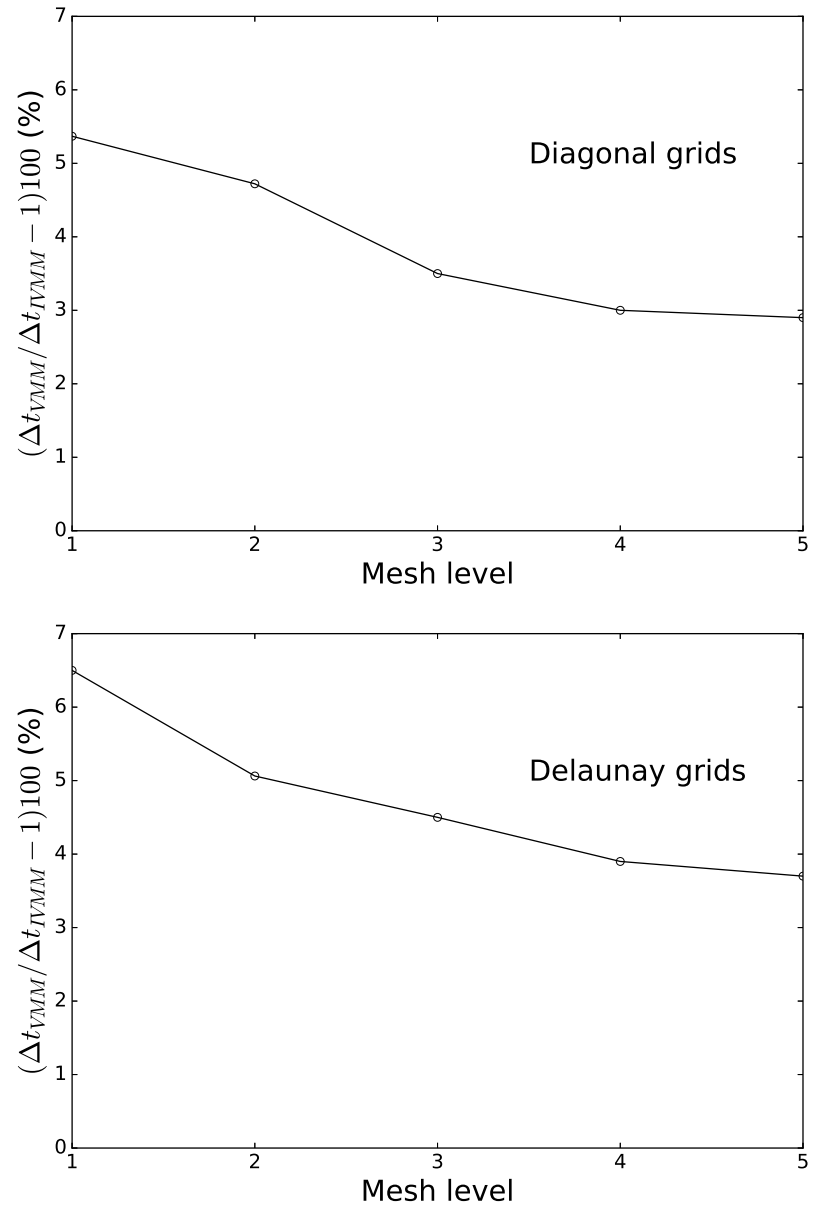

Figure 10: Moving shorelines in a two-dimensional frictional parabolic bowl: relative computational time for a single step for VMM scheme and IVMM scheme for the diagonal grids (upper) and Delaunay grids (lower). 


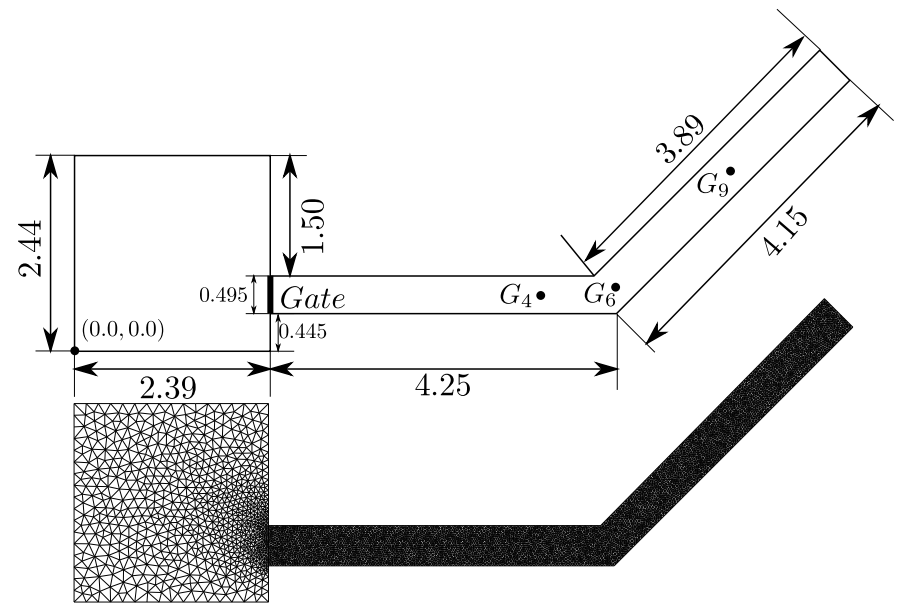

Figure 11: Dam-break in a channel with $45^{\circ}$ bend: plan view of the experiment set up (upper) and the computational grid (lower), values in (m). 

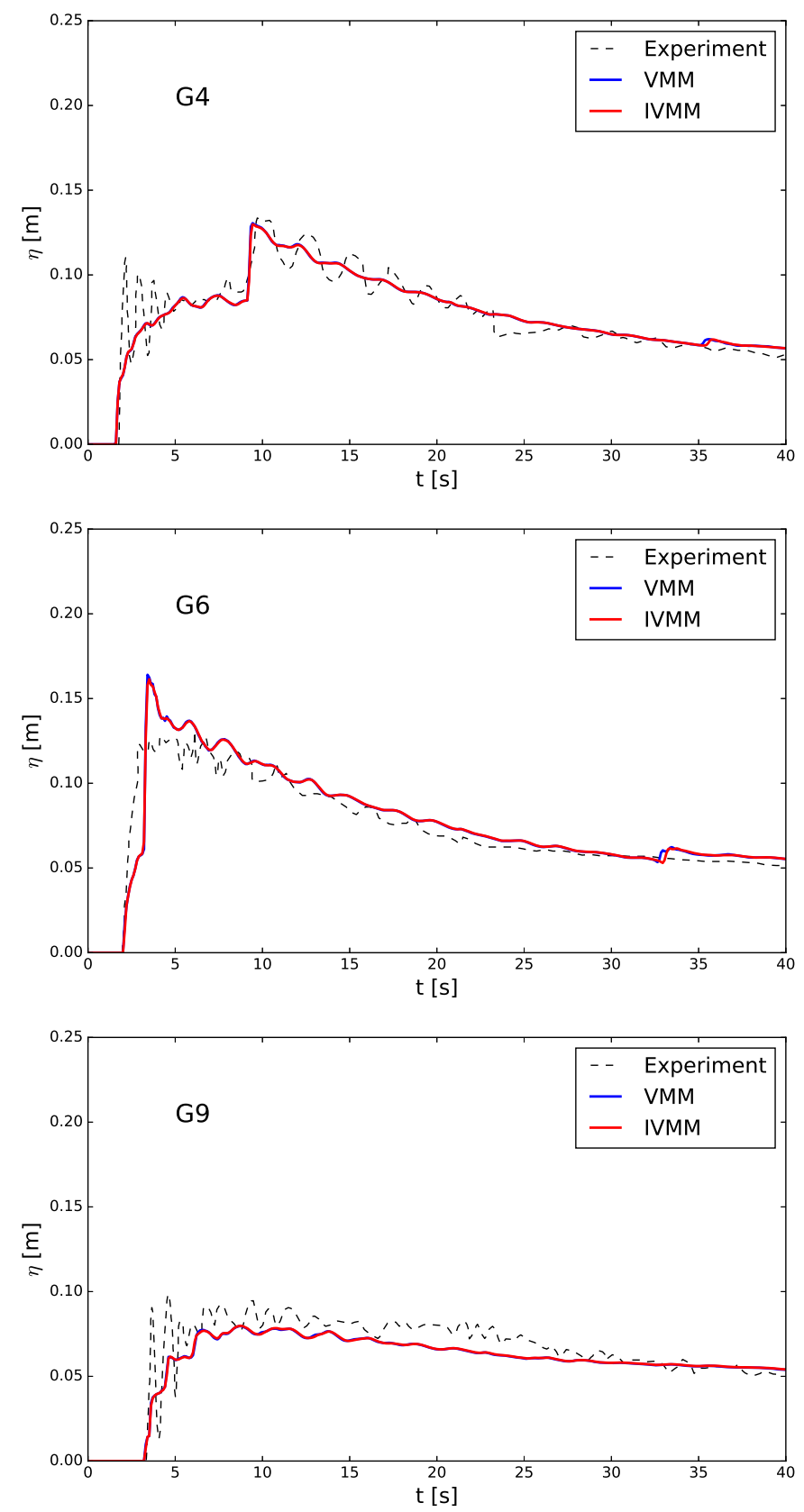

Figure 12: Dam-break in a channel with $45^{\circ}$ bend: water level at G4 (upper), G6 (middle), G9 (lower) against measurements. 


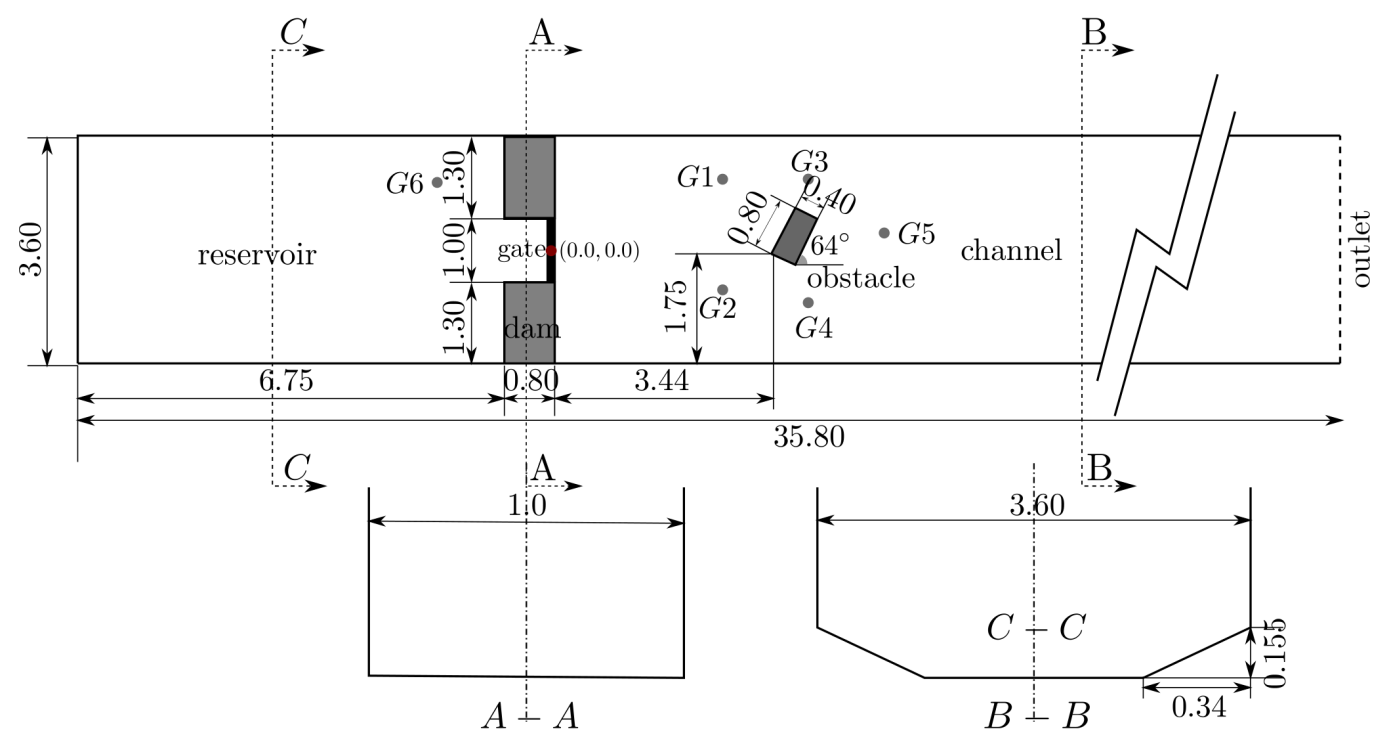

Figure 13: Two-dimensional dam-break flow against an isolated obstacle: sketch of the experiment set up (m) after [33].

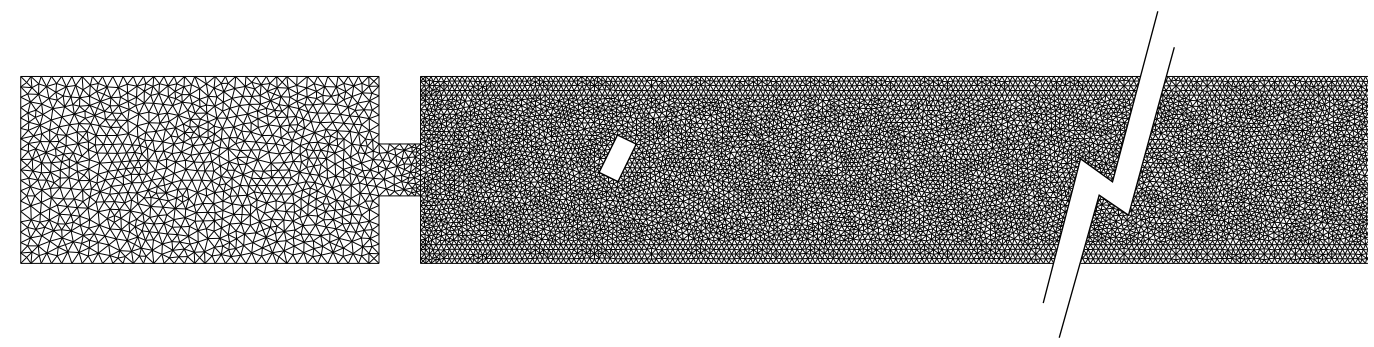

Figure 14: Two-dimensional dam-break flow against an isolated obstacle: computational grid used for simulation. 

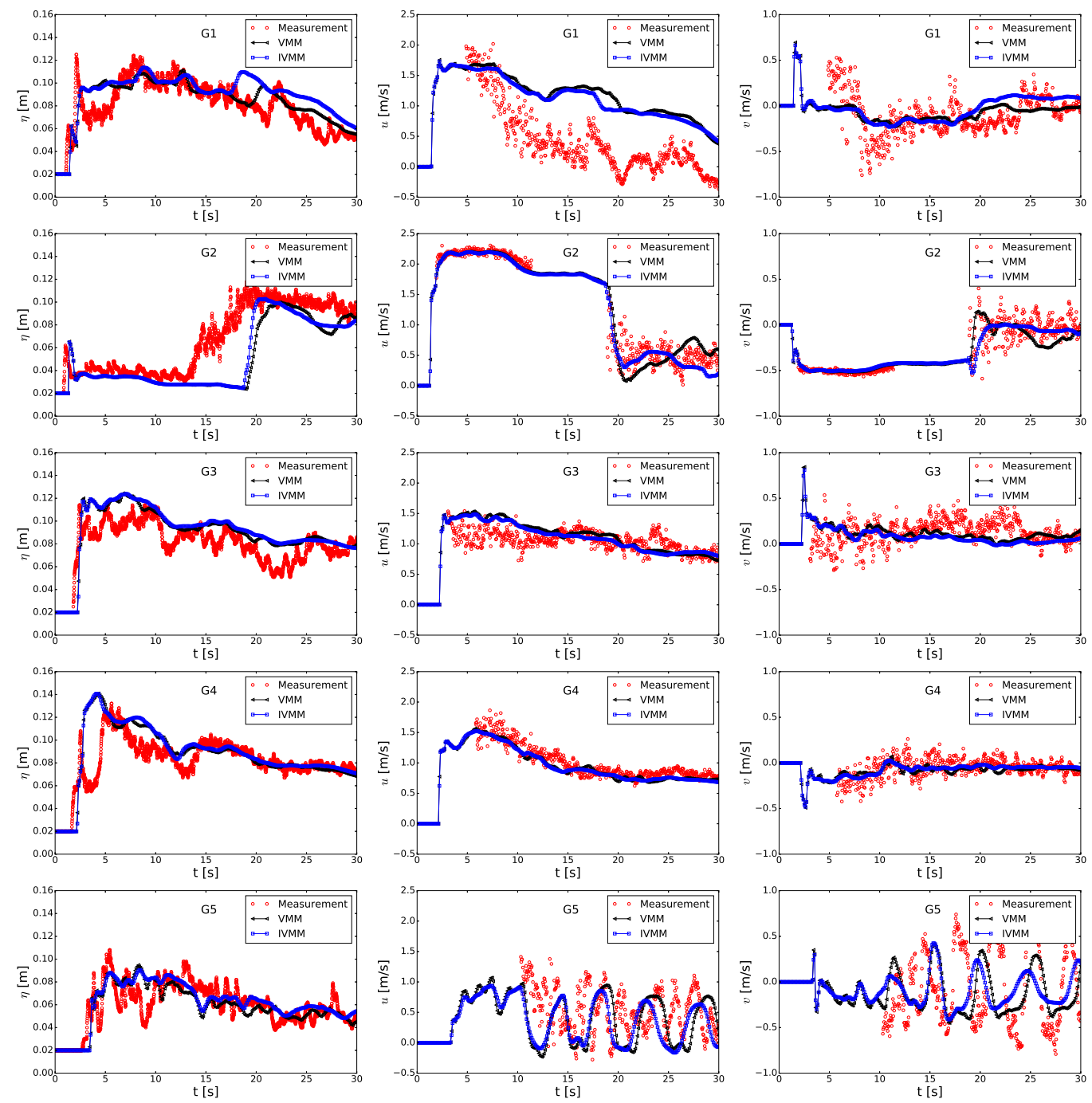

Figure 15: Two-dimensional dam-break flow against an isolated obstacle: the comparison of water elevation (left), the velocity along $x$ - direction (middle) and $y$ - direction (right) between measurement data (००), and simulation results from VMM ( $\square-\square)$ and IVMM $(\triangleleft-\triangleleft)$ at gauges G 1-5. 


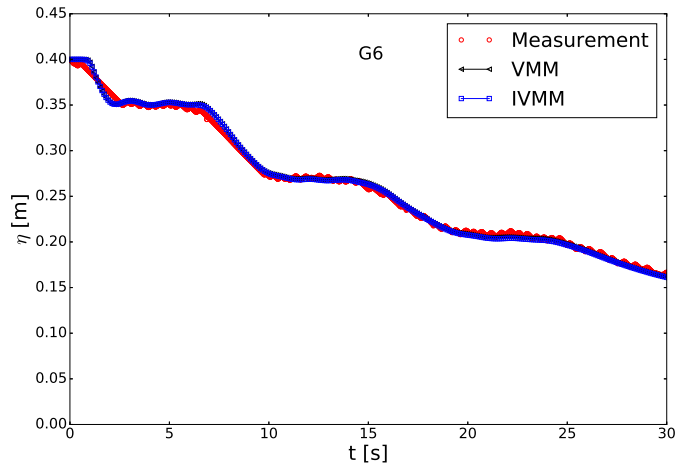

Figure 16: Two-dimensional dam-break flow against an isolated obstacle: the comparison of water elevation between measurement data $(\circ \circ)$, and simulation results from VMM $(\square-\square)$ and IVMM $(\triangleleft-\triangleleft)$ at gauges G 6 . 

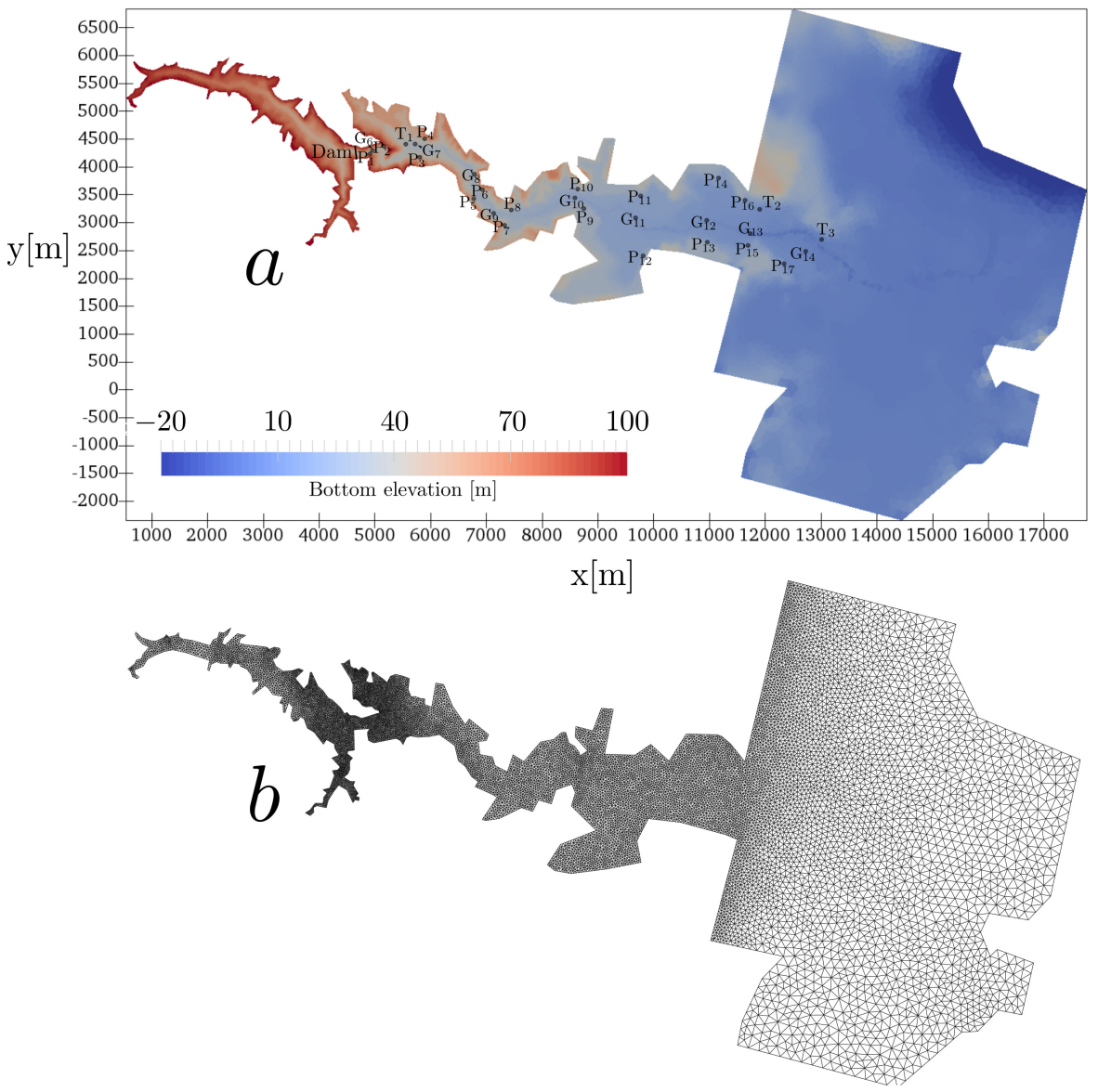

Figure 17: Malpasset dam-break: topography and locations of electrical transformers $T$, survey points $P$ and experimental gauges $\mathrm{G}$ (a); computational domain and simulation grid (b). 


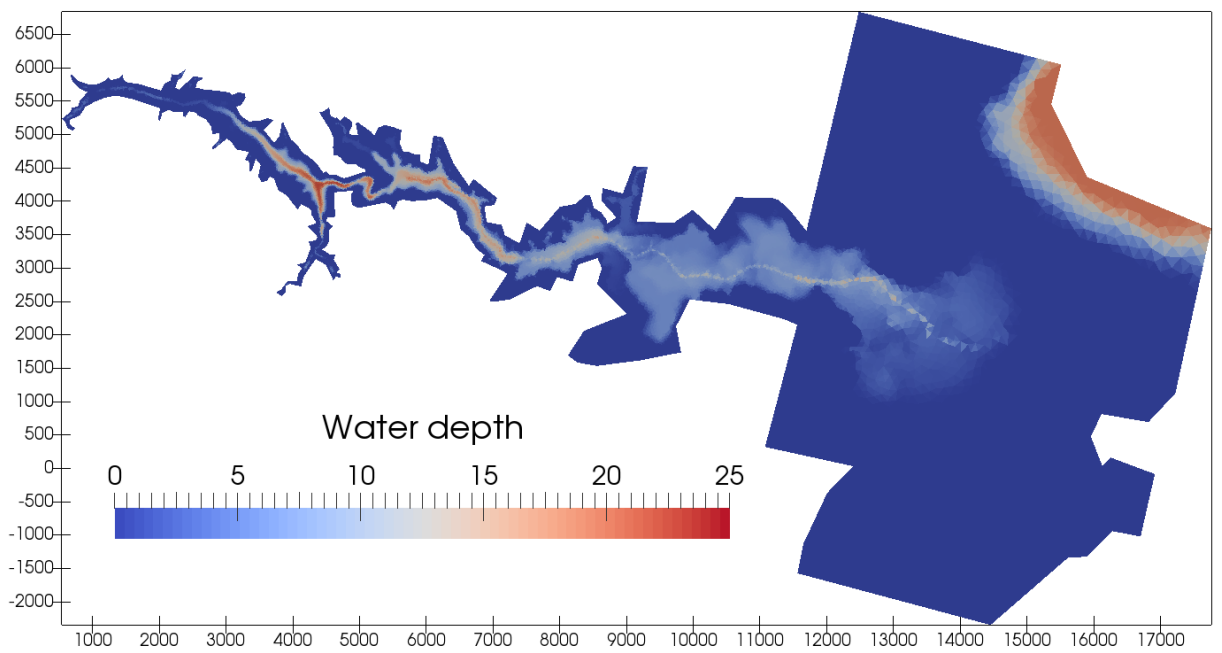

Figure 18: Malpasset dam-break: predicted water depth (m) by IVMM scheme at $t=$ $2000 \mathrm{~s}$. 

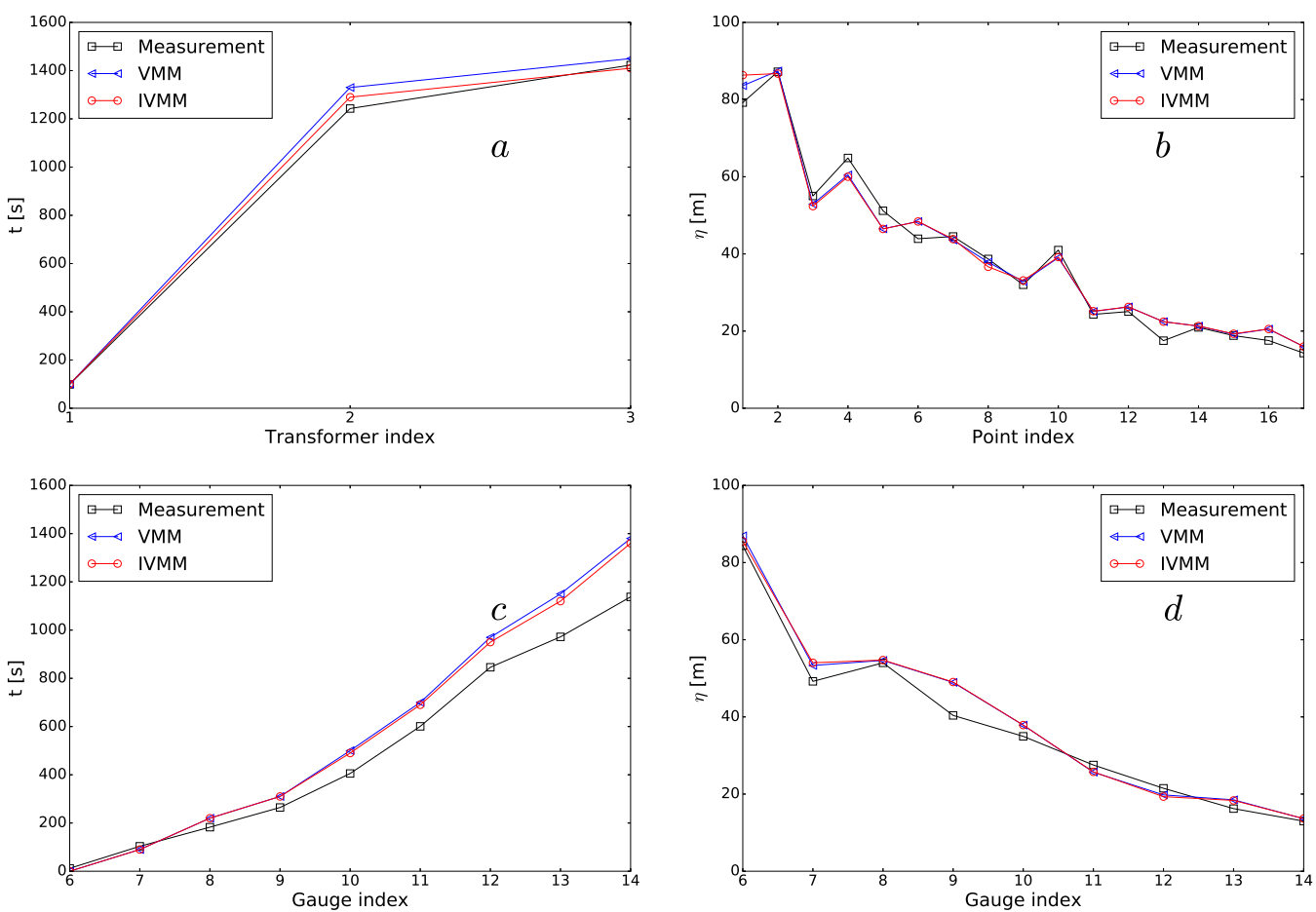

Figure 19: Malpasset dam-break: (a) arrival times at three electrical transformers, (b) maximum water levels at survey points, (c) arrival times at experiment gauges, (d) maximum water levels at experimental gauges. 